\title{
JAK2-IGF1 axis in osteoclasts regulates postnatal growth in mice
}

\author{
David W. Dodington, ${ }^{1}$ Jenalyn L. Yumol, ${ }^{2}$ Jiaqi Yang, ${ }^{1}$ Evan Pollock-Tahiri, \\ Tharini Sivasubramaniyam, ${ }^{1}$ Sandra M. Sacco, ${ }^{2}$ Stephanie A. Schroer, ${ }^{1}$ Yujin E. Li, ${ }^{1}$ Helen Le, ${ }^{1}$ \\ Wendy E. Ward, ${ }^{2,3}$ and Minna Woo ${ }^{1,4,5}$ \\ ${ }^{1}$ Toronto General Hospital Research Institute, University Health Network, Toronto, Ontario, Canada. ${ }^{2}$ Department of \\ Kinesiology and ${ }^{3}$ Department of Health Sciences, Brock University, St. Catharines, Ontario, Canada. ${ }^{4}$ Institute of Medical \\ Science and Department of Immunology and ${ }^{5}$ Division of Endocrinology and Metabolism, Department of Medicine, \\ University Health Network/Sinai Health System, University of Toronto, Toronto, Ontario, Canada.
}

\begin{abstract}
Osteoclasts are specialized cells of the hematopoietic lineage that are responsible for bone resorption and play a critical role in musculoskeletal disease. JAK2 is a key mediator of cytokine and growth factor signaling; however, its role in osteoclasts in vivo has yet to be investigated. To elucidate the role of JAK2 in osteoclasts, we generated an osteoclast-specific JAK2-KO (OcJAK2-KO) mouse using the Cre/Lox-P system. Oc-JAK2-KO mice demonstrated marked postnatal growth restriction; however, this was not associated with significant changes in bone density, microarchitecture, or strength, indicating that the observed phenotype was not due to alterations in canonical osteoclast function. Interestingly, Oc-JAK2-KO mice had reduced osteoclast-specific expression of ICF1, suggesting a role for osteoclast-derived IGF1 in determination of body size. To directly assess the role of osteoclast-derived IGF1, we generated an osteoclast-specific ICF1-KO mouse, which showed a similar growth-restricted phenotype. Lastly, overexpression of circulating IGF1 by human transgene rescued the growth defects in Oc-JAK2-KO mice, in keeping with a causal role of IGF1 in these models. Together, our data show a potentially novel role for Oc-JAK2 and IGF1 in the determination of body size, which is independent of osteoclast resorptive function.
\end{abstract}

Conflict of interest: The authors have declared that no conflict of interest exists.

Copyright: (c) 2021, Dodington et al. This is an open access article published under the terms of the Creative Commons Attribution 4.0 International License.

Submitted: February 5, 2020 Accepted: January 20, 2021 Published: March 8, 2021

Reference information: /CI Insight. 2021;6(5):e137045.

https://doi.org/10.1172/jici. insight.137045.

\section{Introduction}

Osteoclasts are specialized cells of the hematopoietic lineage and mostly known for their function in bone resorption (1). Osteoclasts play a critical role in the pathogenesis of several diseases, including osteoporosis (2), rheumatoid arthritis (3), periodontal disease (4), myeloma (5), and metastatic cancer (6); thus, there is an increasing need to elucidate the mechanisms of osteoclast function under homeostatic conditions in order to better understand the pathogenesis of osteoclast-related diseases and develop novel therapeutic strategies.

Janus kinase 2 (JAK2) is a ubiquitously expressed tyrosine kinase involved in multiple signal transduction pathways (7). JAK2 is responsible for transmitting extracellular signals from various cytokines and growth factors, including growth hormone, erythropoietin, thrombopoietin, prolactin, IL-6, granulocyte-macrophage CSF (GM-CSF), and IFN- $\gamma$. JAK2 is also a clinically relevant gene due to the causal link between activating JAK2 mutations and myeloproliferative disorders (8). In bone, there is some evidence to suggest a role for JAK2 in osteoclast biology. Osteoclasts derived from individuals with myelofibrosis harboring JAK2 activating mutations have impaired osteolytic capacity, implicating JAK2 with osteoclast dysfunction (9). In vitro studies using JAK2 inhibitors have also highlighted a potential role for JAK2 in osteoclasts. Osteoblast/osteoclast cocultures treated with the JAK1/2 inhibitor baricitinib showed decreased osteoclastogenesis due to reduced RANKL expression in osteoblasts (10). In another study, use of the JAK2 inhibitor AG490 in purified osteoclast cultures inhibited osteoclast apoptosis in response to RANKL withdrawal (11). Despite these data, the role of osteoclast JAK2 in vivo has yet to be investigated.

The objective of this study was to elucidate the in vivo function of JAK2 in osteoclasts. To accomplish this, we used the Cre/Lox-P system to generate an osteoclast-specific JAK2-KO (Oc-JAK2-KO) mouse. In this study, we found that Oc-JAK2-KO mice had marked postnatal growth restriction; however, this was not associated with significant changes in bone mineral density (BMD), bone microarchitecture, bone strength, osteoclast number, or osteoclastic gene expression, suggesting that osteoclast JAK2 modulates 
body size without altering the balance between bone formation and bone resorption. Interestingly, loss of JAK2 impaired expression of IGF1 in osteoclasts, suggesting an important role for osteoclast-derived IGF1 in determination of body size. We also show that overexpression of circulating IGF1 in Oc-JAK2$\mathrm{KO}$ mice rescued their growth defects, supporting a causal role between JAK2-mediated IGF1 expression in osteoclasts and postnatal growth.

\section{Results}

Generation of Oc-JAK2-KO mice. To validate the osteoclast specificity of the Cathepsin K-Cre (Ctsk-Cre) mouse, we bred $C t s k$-Cre ${ }^{+}$mice to $m \mathrm{Tm}^{+/-}$reporter mice whose tissue expressed either fluorescence TdTomato in the absence of Cre-mediated recombination or enhanced GFP (EGFP) in its presence. Sections of the knee joint showed robust expression of EGFP along the surface of the trabecular bone in $\mathrm{Ctsk}_{-\mathrm{Cre}}^{+} m \mathrm{C}_{-}$ $m G^{+}$mice (Figure 1A). Staining for tartrate resistant alkaline phosphatase (TRAP) on the adjacent serial section confirmed that EGFP was present in the expected distribution for osteoclasts. EGFP was not detected in $\mathrm{Cre}^{-}$controls. Sections of other tissues, including growth plate cartilage, brain, hypothalamus, testis, ovary, pancreas, liver, small intestine, kidney, and thyroid did not express EGFP (Figure 1B). Next, to generate Oc-JAK2-KO mice, Ctsk-Cre ${ }^{+}$mice were bred to Jak2 floxed mice (Jak2 $2^{f / f f}$ ) and resultant Ctsk-Cre ${ }^{+-}$

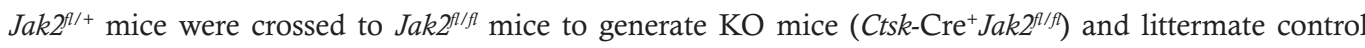
mice $\left(J a k 2^{f / f l}\right.$ and $\left.C t s k-C r e^{+} J a k 2^{f /+}\right)$. To validate osteoclast-specific deficiency of JAK2 in our Oc-JAK2-KO mouse, we measured Jak2 mRNA levels in tissues and in BM-derived osteoclasts from Ctsk-Cre ${ }^{+} J a 2^{f / f l}$ and $J a k^{f l f l}$ mice. JAK2 mRNA expression was reduced by $26 \%$ in bone and by $70 \%$ in osteoclast cultures from Ctsk-Cre ${ }^{+} J a k 2^{f l / l}$ mice (Figure 1C). Importantly, Jak2 mRNA expression was unchanged in BM-derived macrophages, liver, testis, ovary, or hypothalamus. There have been previous reports of Ctsk-Cre mediating germline gene deletion of certain floxed alleles (12); however, this did not occur in Ctsk-Cre $J a k 2^{f / f l}$ mice, since Jak2 expression was preserved in nonosteoclast cells and tissues. Lastly, we performed IHC for JAK2 on femur sections with TRAP staining on the adjacent serial section to identify $\mathrm{TRAP}^{+}$multinucleated giant cells. In control mice, there was weak to moderate immunostaining for JAK2 in TRAP ${ }^{+}$multinucleated giant cells (Figure 1D). In Ctsk-Cre $J a k 2^{f / f l}$ mice, immunoreactivity for JAK2 was absent in TRAP multinucleated giant cells, with retained expression in other BM cells. Overall, these results indicate that Ctsk-Cre $J a k 2^{f l / f l}$ mice represent a rigorous experimental model to study the role of osteoclast JAK2 in vivo. For all experiments, both $J a k 2^{f / f l}$ and $C t s k-C r e^{+} J a k 2^{f /+}$ mice were used as controls in approximately equal proportions unless otherwise specified. There were no significant differences in any measured outcomes between $J a k 2^{f / f l}$ and $C t s k-C r e^{+} J a k 2^{f /+}$ mice, indicating that phenotypic differences observed were not to due Cre expression, nor were there any gene dosage effects present with haploinsufficient Jak2.

Ctsk-Cre Jak2 $2^{f / f l}$ mice demonstrate postnatal growth restriction. Control and Ctsk-Cre ${ }^{+} J a k 2^{f / f l}$ mice were born in the expected Mendelian ratio and appeared normal at birth, with similar body weights up to 1 month of age. By 4 weeks of age in males (Figure 2A), and 5 weeks of age in females (Figure 2B), Ctsk-Cre ${ }^{+} J a k 2^{f / f l}$ mice began to exhibit significantly lower body weight compared with littermate controls. There were no grossly identifiable abnormalities, aside from reductions in overall body size in Ctsk-Cre ${ }^{+} J a k 2^{f / f l}$ mice (Figure $2 \mathrm{C}$ ). Measurement of total body length showed that body length was similar at 2 weeks of age, but a clear distinction in body length between control and $C t s k-C r e{ }^{+} J a k 2^{f / f l}$ mice was observed at 8 and 24 weeks of age (Figure 2, D and E). Similarly, there was an approximate 10\% reduction in femur length in both male and female Ctsk-Cre $J a k 2^{f l f l}$ mice compared with littermate controls at 8 and 24 weeks, but not at 2 weeks of age (Figure 2, F-H). Body composition analysis is shown in Table 1. Weights of bone, skeletal muscle, and internal organs were frequently reduced in $\mathrm{Ctsk}-\mathrm{Cre}{ }^{+} \mathrm{Jak} 2^{f / f l}$ mice but did not show any significant differences when normalized to body length. Overall, Ctsk-Cre $J a k 2^{f l f l}$ mice demonstrate proportional reduction in body size in the postnatal period.

Deletion of JAK2 in osteoclasts does not alter bone homeostasis. Next, we sought to determine if targeted deletion of JAK2 in osteoclasts resulted in changes in bone health or osteoclast biology. First, we performed Dual-energy x ray absorptiometry (DXA) scan on excised femurs and lumbar vertebrae (LV). Both male and female Ctsk-Cre $J a k 2^{f / f l}$ mice had reduced femoral BMD at 24 weeks of age compared with littermate controls; however, this difference was no longer significant after adjustment for body length (Figure 3, A and B). Since measurement of areal BMD underestimates bone density in the setting of short stature or growth delay, we applied the principles from the Pediatric Official Positions statement (International society of Clinical Densiometry), which recommend adjusting areal BMD to height (13). 
A
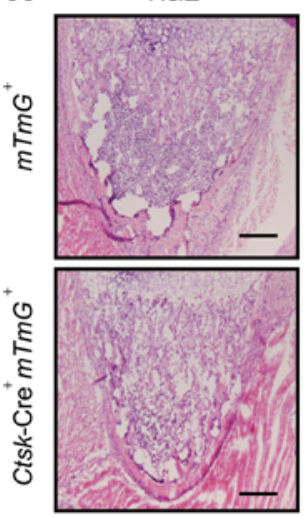

B

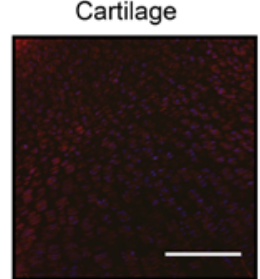

Pancreas

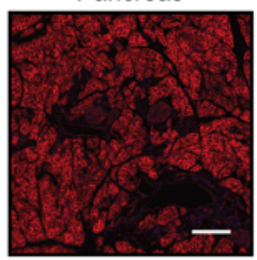

TRAP
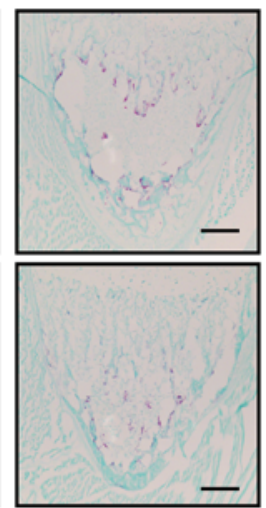

Brain

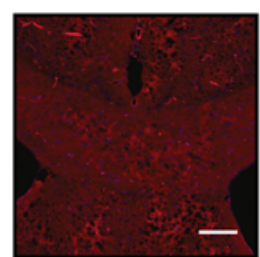

Liver \& Gallbladder

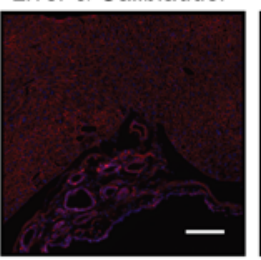

TdTomato
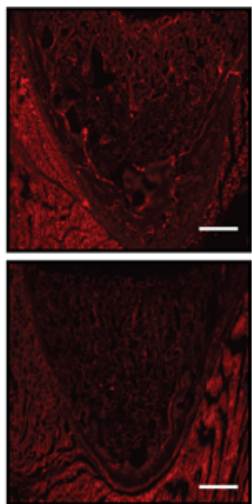

Hypothalamus

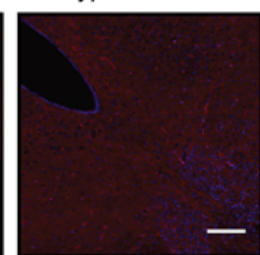

Intestine

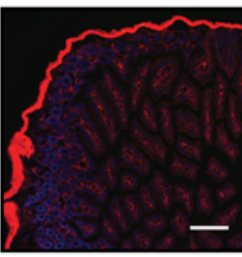

GFP
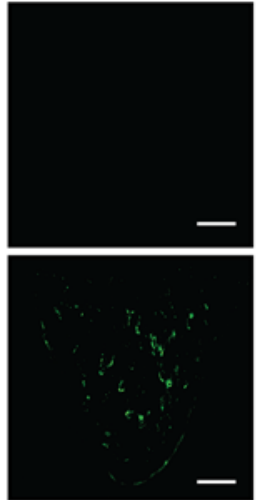

Testis

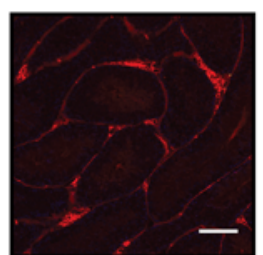

Kidney

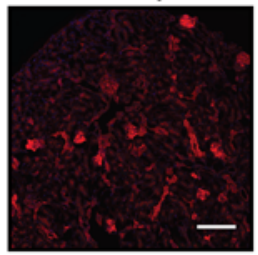

TdTomato/GFP/DAPI
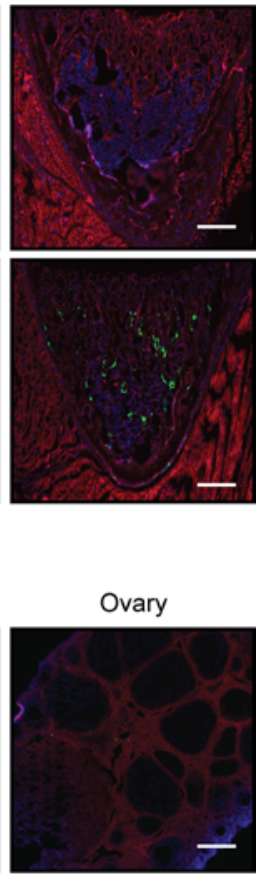

Thyroid

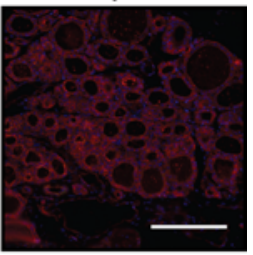

C

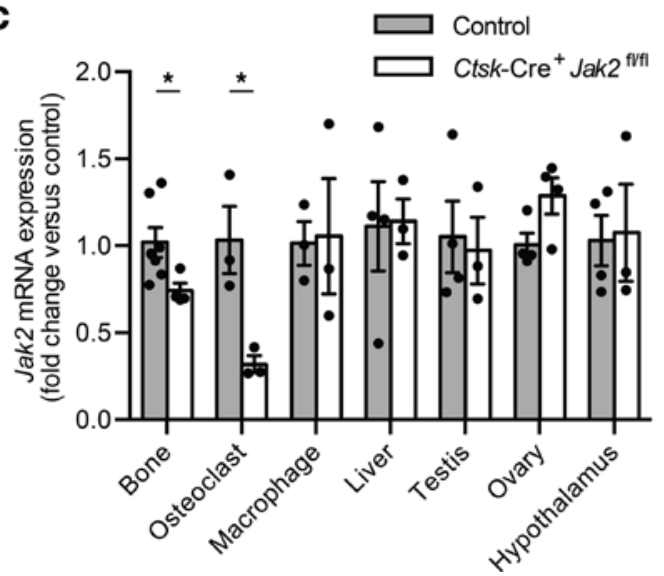

D
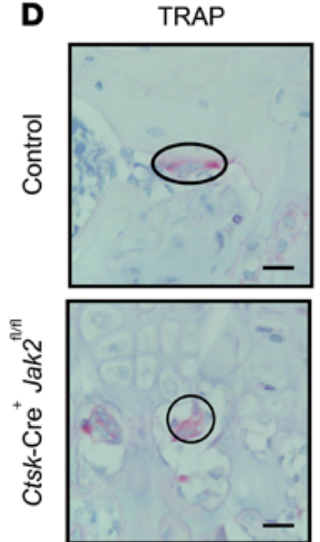

JAK2
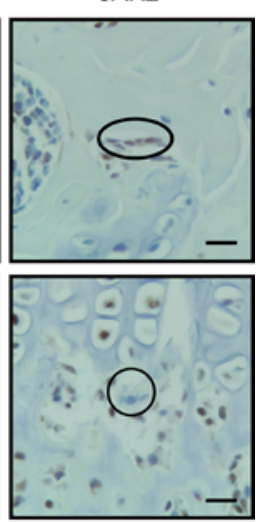

Figure 1. Generation of osteoclast-specific JAK2-KO mice. Ctsk-Cre-expressing mice were bred to $m T m G$ mice or mice with floxed Jak2 gene to generate reporter and osteoclast-specific JAK2-KO mice. (A) Representative images of knee joint from $m \operatorname{TmG}^{+}(n=3)$ and Ctsk-Cre $m \mathrm{CTm}^{+}$mice $(n=3)$ at 1 week of age stained for $\mathrm{H \& E}$, tartrate resistant alkaline phosphatase (TRAP), and imaged for fluorescence of endogenous Cre reporter activity. Staining/imaging was performed on adjacent serial frozen sections. Scale bars: $100 \mu \mathrm{m}$. (B) Representative fluorescence images of cartilage (femur growth plate), brain, hypothalamus, testis, ovary, pancreas, liver/gallbladder, small intestine, kidney, and thyroid from Ctsk-Cre+ $m T_{m} G^{+}$mice $(n=3)$. Tissues were collected from 8-week-old mice, except for growth

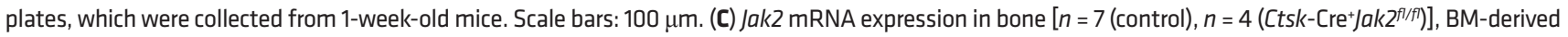
osteoclasts $\left[n=3\right.$ (control), $n=3\left(\right.$ Ctsk-Cre+ $\left.\left.J a k 2^{f / f}\right)\right]$, BM-derived macrophages $\left[n=3\right.$ (control), $n=3\left(\right.$ Ctsk-Cre $\left.\left.{ }^{+} J a k 2^{f / f}\right)\right]$, liver $\left[n=4\right.$ (control), $n=3\left(\right.$ Ctsk-Cre $\left.\left.{ }^{+} J a k 2^{f / f f}\right)\right]$, testis $\left[n=4\right.$ (control), $n=3$ (Ctsk-Cre $\left.\left.J a k 2^{f / f}\right)\right]$, ovary $\left[n=4\right.$ (control), $n=4($ Ctsk-Cre $\left.\left.) a k 2^{f / f}\right)\right]$, and hypothalamus $[n=4$ (control), $n=3$ (Ctsk-Cre+ $\left.\left.) a k 2^{f / f f}\right)\right]$ from control and [tsk-Cre ${ }^{+}$ak $2^{\text {flflf }}$ mice. Values are normalized to 185 mRNA levels and presented as fold change over control group. (D) Representative images of femur from control $(n=3)$ and Ctsk-Cre+Jak $2^{f / f l}$ mice $(n=3)$ stained for TRAP and immunostained for JAK2. Staining was performed on adjacent serial sections. The circle highlights TRAP ${ }^{+}$multinucleated giant cells. Scale bars: $10 \mu \mathrm{m}$. Data represent mean $\pm \mathrm{SEM}$. Differences between groups were analyzed for statistical significance by Student's unpaired $t$ test; ${ }^{*} P<0.05$. Oval highlights TRAP+ multinucleated giant cells. 
A

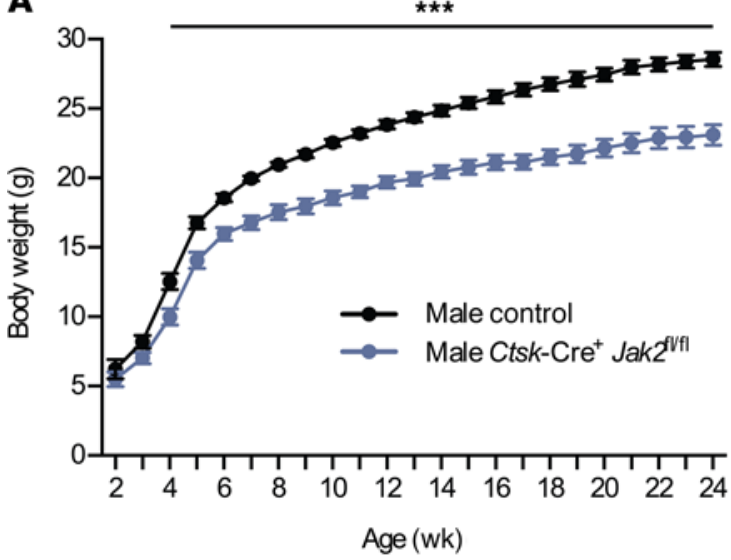

B

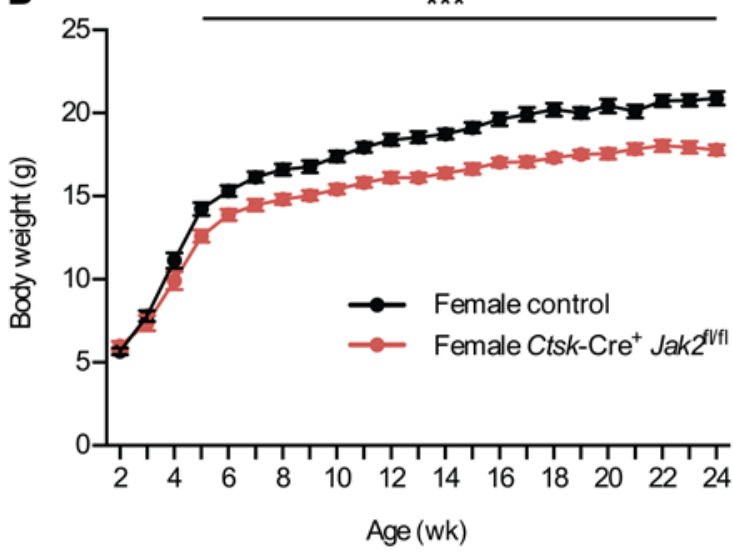

C

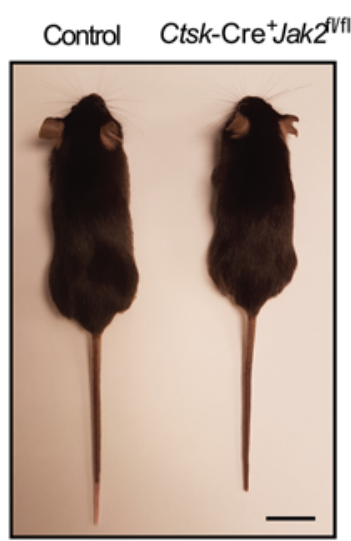

$\mathbf{F}$

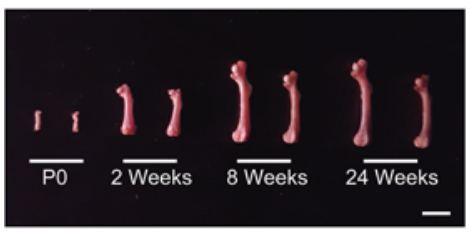

D $\square$ Male control

Male Ctsk-Cre'Jak2 $2^{\text {vif }}$

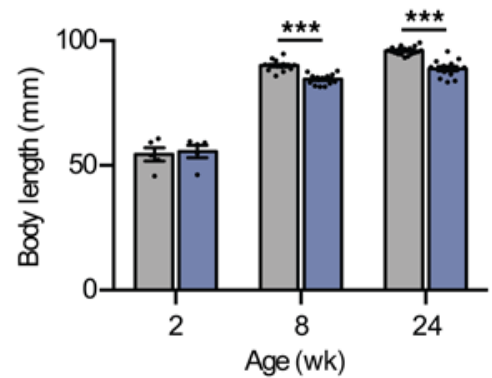

G

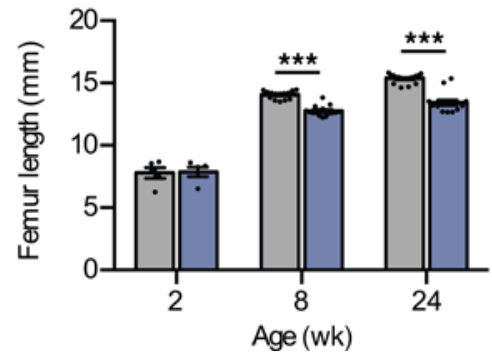

E

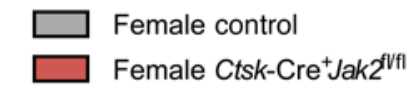

H

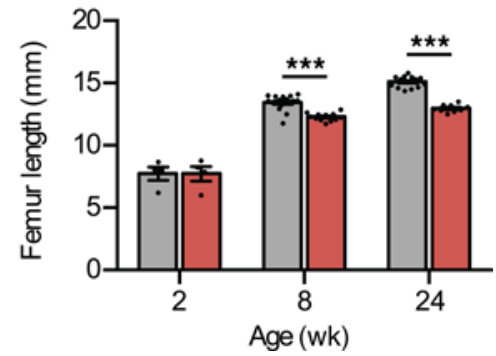

Figure 2. Ctsk-Cre $J a k 2^{f / / f l}$ mice demonstrate postnatal growth restriction. Ctsk-Cre $\int a k 2^{f / f l}$ and littermate control mice were monitored until 6 months of age and growth parameters assessed. (A) Weekly body weights of male control $(n=18)$ and $C t s k$-Cre+ $J a k 2^{f / f l}(n=14)$ mice. (B) Weekly body weights of female control $(n=15)$ and Ctsk-Cre+Jak $2^{f / f l}(n=9)$ mice. (C) Representative photograph of 8-week-old male control (left, $\left.n=16\right)$ and Ctsk-Cre+ $J a k 2^{f / f l}$ (right, $\left.n=15\right)$ mice. Scale bar: $20 \mathrm{~mm}$. (D) Total body length of male control [ $n=5$ (2 weeks), $n=16$ (8 weeks), $n=18$ (24 weeks)] and Ctsk-Cre ${ }^{+} / a k 2^{f / f l}[n=5$ ( 2 weeks), $n=15$ ( 8 weeks), $n$ $=14$ (24 weeks)] mice. (E) Total body length of female control $\left[n=4\right.$ (2 weeks), $n=18$ (8 weeks), $n=15$ (24 weeks)] and Ctsk-Cre $/ a k 2^{f / f f l}[n=4$ ( 2 weeks), $n=12$ (8 weeks), $n=9$ (24 weeks)] mice. (F) Representative photograph of femurs from control (left) $[n=3$ (P0), $n=9$ (2 weeks), $n=34$ (8 weeks), $n=33$ (24 weeks)] and

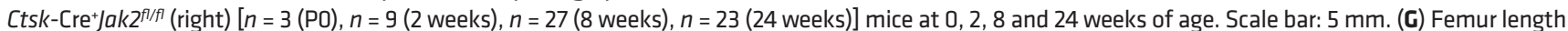

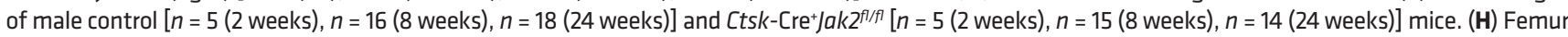
length of female control [ $n=4$ ( 2 weeks), $n=18$ (8 weeks), $n=15$ (24 weeks)] and Ctsk-Cre+Jak $2^{f / f f}[n=4$ (2 weeks), $n=12$ (8 weeks), $n=9$ (24 weeks)] mice. Data represent mean \pm SEM. Differences between groups were analyzed for statistical significance by repeated measures ANOVA or 2 -way ANOVA; ${ }^{* * *} P<0.001$.

At the lumbar spine, there were no significant differences in BMD at LV1-3 both before and after adjusting for body length (Figure 3, C and D).

Bone microarchitecture of femurs from 8-week-old and 24-week-old female mice was then assessed by $\mu \mathrm{CT}$. At the femur neck, there were no changes in trabecular bone measures, including bone volume fraction and trabecular number, thickness, or separation (Figure 4A). At the distal femur, $\mathrm{Ctsk}-\mathrm{Cre}^{+} \mathrm{Jak} 2^{\mathrm{fl} / \mathrm{l}}$ mice had reduced trabecular thickness at 8 and 24 weeks, but there were no changes in trabecular number, separation, or overall bone volume fraction (Figure 4B). At the femur midpoint, Ctsk-Cre $J a k 2^{f / f l}$ mice 
Table 1. Body composition of Ctsk-Cre+Jak2 ${ }^{f 1 / f l}$ mice $^{A}$

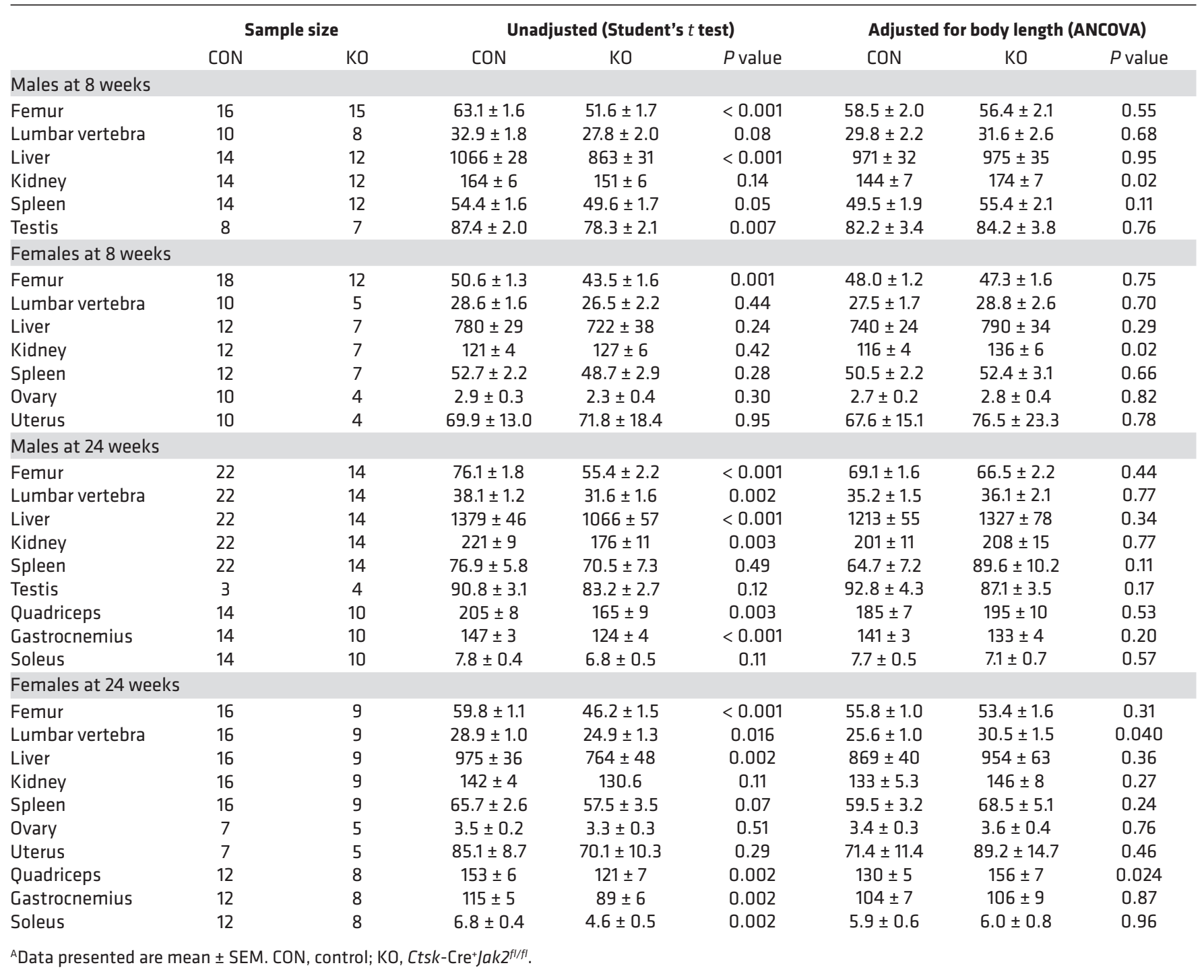

showed reduction in total area and cortical area; however, the cortical area/total area ratio and cortical thickness were unchanged (Figure 4C). Overall, the $\mu \mathrm{CT}$ data show some minor changes in bone microarchitecture, which are likely attributable to the differences in body size of $C t s k-C r e^{+} J a k 2^{f l f l}$ mice.

Next, we assessed biomechanical strength of femurs and LV using a materials testing system. Threepoint bending at the femur midpoint showed no difference in peak load both before and after adjustment for body size (Figure 5, A and B). At the femur neck, both male and female Ctsk-Cre $\mathrm{Jak}^{\mathrm{fl} / f l}$ mice had reduced compressive peak load at 24 weeks of age; however, this was no longer significant after adjustment for body size (Figure 5, C and D). At the lumbar spine, there was a nonsignificant trend toward reduced compressive peak load in $\mathrm{Ctsk}-\mathrm{Cre}^{+} \mathrm{Jak} 2^{f / f l}$ mice, which did not persist after adjustment for body size (Figure 5, E and F). Forelimb grip strength was also measured using a rodent grip strength meter. Male Ctsk-Cre ${ }^{+} J a k 2^{f / f l}$ mice had reduced grip strength at 8 and 24 weeks, while female $C t s k-C r e^{+} J a k 2^{f / f t}$ mice demonstrated reduced grip strength at 24 weeks; however, these differences did not persist after adjustment for body size (Figure 5, G and H). Overall, the biomechanical strength testing shows reductions in musculoskeletal strength in $\mathrm{Ctsk}-\mathrm{Cre}{ }^{+} \mathrm{Jak} 2^{f / f l}$ mice that are proportional to body size.

Next, we assessed for effects of JAK2 deficiency on osteoclast parameters. TRAP stain to identify osteoclasts in femur sections showed no alteration in osteoclast numbers or distribution in $\mathrm{Ctsk}_{\mathrm{Cre}} \mathrm{Cak}^{\mathrm{f} / / \mathrm{fl}}$ mice (Figure 6A). Expression of key genes involved in osteoclastogenesis and osteoclast function were 

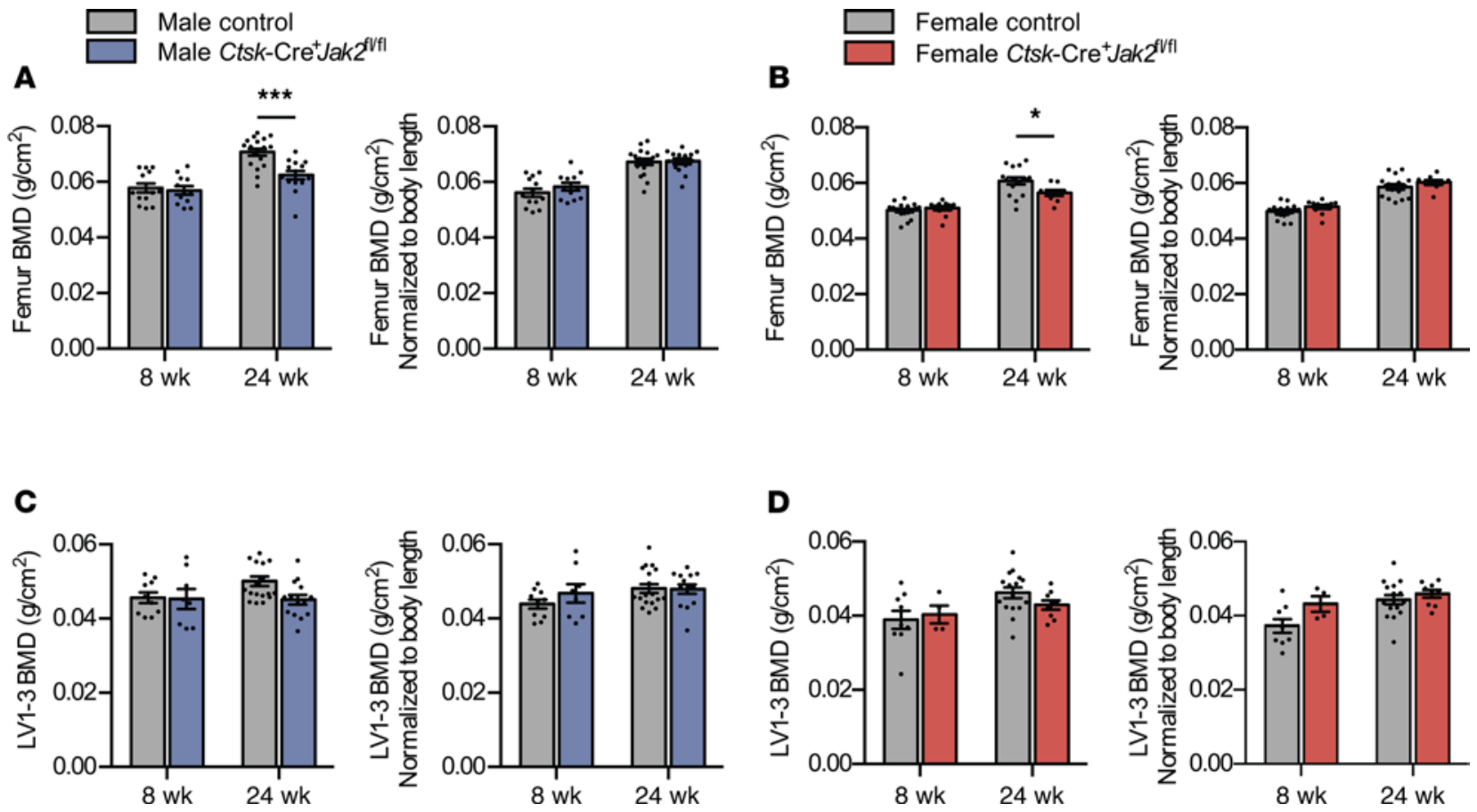

Figure 3. Ctsk-Cre+Jak2 $2^{f / f l}$ mice have proportional reduction in bone mineral density. Excised femurs and lumbar vertebrae 1-3 (LV1-3) from 8- and 24-week-old Ctsk-Cre ${ }^{+} a k 2^{f l / f l}$ and control mice were analyzed by dual x ray absorptiometry (DXA). (A) Femur bone mineral density (BMD) (left panel) and BMD normalized to body length (right panel) in male control [ $n=13$ (8 weeks), $n=18$ (24 weeks)] and Ctsk-Cre ${ }^{+} / a k 2^{f / f l}[n=12$ ( 8 weeks), $n=14$ (24 weeks)] mice. (B) Femur BMD (left panel) and BMD normalized to body length (right panel) in female control [ $n=18$ (8 weeks), $n=16$ (24 weeks)] and Ctsk-Cre ${ }^{+}$ak $2^{f l / f l}[n=12$ (8 weeks), $n=9$ (24 weeks)] mice. (C) Lumbar vertebrae 1-3 (LV1-3) BMD (left panel) and BMD normalized to body length (right panel) in male control [ $n=10$ (8 weeks), $n=18$ (24 weeks)] and Ctsk-Cre+Jak2 fl/fl $[n=8$ (8 weeks), $n=14$ (24 weeks)] mice. (D) LV1-3 BMD (left panel) and BMD normalized to body length (right panel) in female control $\left[n=9\right.$ ( 8 weeks), $n=16$ (24 weeks)] and Ctsk-Cre ${ }^{+} J a k 2^{f / f l}[n=4$ (8 weeks), $n=9$ (24 weeks)] mice. Data represent mean \pm SEM. Differences between groups were analyzed for statistical significance by 2-way ANOVA; ${ }^{*} P<0.05$, ${ }^{* *} P<0.001$.

quantified in whole bone samples from the distal femur and proximal tibia (Figure 6B). There were no differences in expression of calcitonin receptor (Calcr), tartrate-resistant acid phosphatase type 5 (Acp5), Ctsk, osteoclast-associated receptor (Oscar), integrin $\beta 3$ (Itgb3), matrix metalloproteinase 9 ( $\mathrm{Mmp} 9$ ), C-terminal Src kinase (Csk), and nuclear factor of activated t cells 1 ( Nfatc1).

Growth plates were then evaluated on Safranin O-stained sections of tibia (Figure 6C). At 2 weeks of age, there were no differences in the size of the growth plate (Figure 6D); however, at 8 weeks of age, Ctsk-Cre $J a k 2^{f l / l}$ mice demonstrated a significant reduction in the size of the zone of hypertrophy $(\mathrm{ZH})$ compared with control mice but not in the size of the total growth plate, zone of proliferation (ZP), or zone of ossification (ZO) (Figure 6E).

Overall, these data show that deletion of JAK2 in osteoclasts does not have a positive or negative effect on bone density, bone microarchitecture, biomechanical strength, osteoclast number, or expression of osteoclastogenic genes. Histologically, there was a reduction in the size of the $\mathrm{ZH}$ in $\mathrm{Ctsk}-\mathrm{Cre}{ }^{+} J a k 2^{f / f t}$ mice in association with their attenuation in body growth.

Reduced osteoclast-specific expression of IGF1 in Ctsk-Cre $J a k 2^{f l / f l}$ mice. Growth defects in osteoclast-specific $\mathrm{KO}$ mice often arise due to impaired osteoclast function and an inability to resorb bone at the developing growth plate (14-15). Despite clear growth defects in $C t s k-C r e^{+} J a k 2^{f l f l}$ mice, there was surprisingly no evidence of defects in osteoclast function. Thus, we sought to investigate an alternative mechanism to explain the growth defects observed in $\mathrm{Ctsk}-\mathrm{Cre}^{+} \mathrm{Jak} 2^{\text {fl/fl}}$ mice.

Since Oc-JAK2-KO led to overall proportional changes in body size and reductions in the growth plate $\mathrm{ZH}$, we hypothesized that a secreted growth factor might play an important role. JAK2 is a wellestablished regulator of IGF1 expression (16-19); therefore, we assessed IGF1 levels in Ctsk-Cre ${ }^{+}$Jak2 $^{\text {flfl }}$ mice. Serum IGF1 concentrations, measured by ELISA, were equivalent in control and $C t s k-\mathrm{Cre}^{+} J a k 2^{f / f l}$ mice at 8 weeks of age (Figure 7A). Quantitative PCR (qPCR), however, showed a decrease in IgfI mRNA expression in the distal femur/proximal tibia in Ctsk-Cre ${ }^{+} J a k 2^{f / f l}$ compared with controls, while no changes in IgfI expression in other tissues - including the liver, testis, and ovary - were present 
A

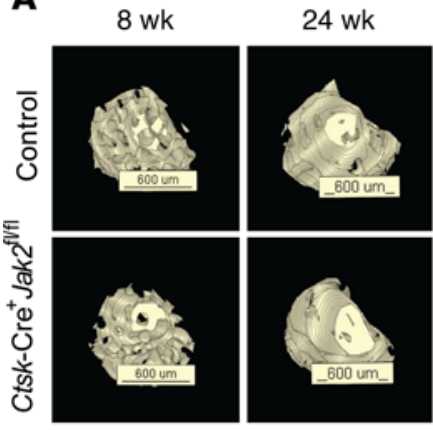

B
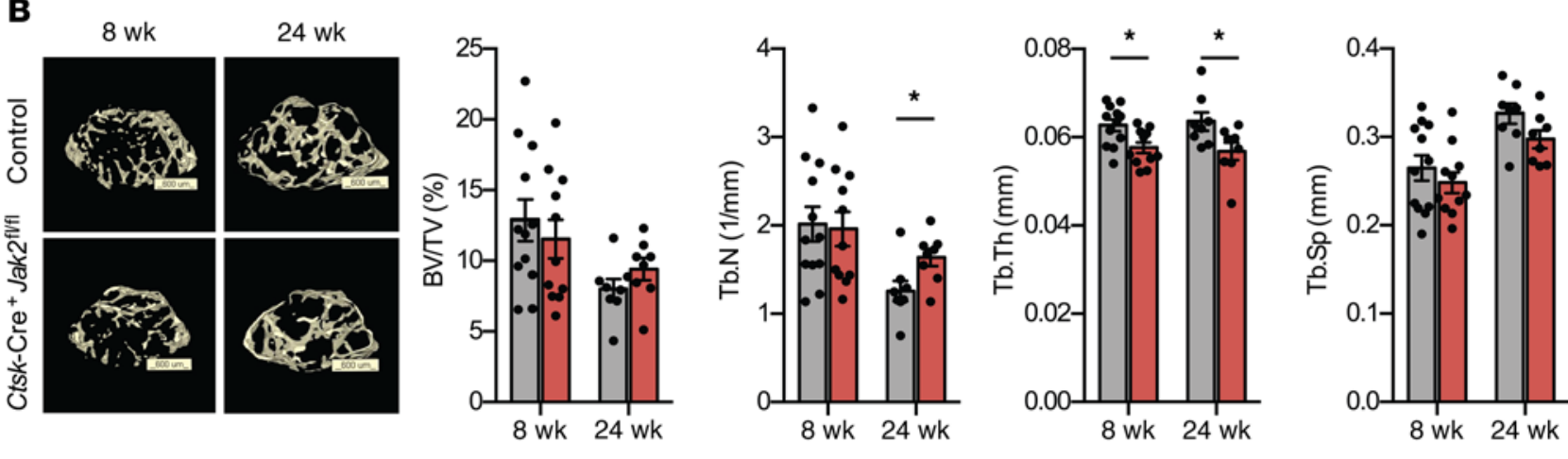

C
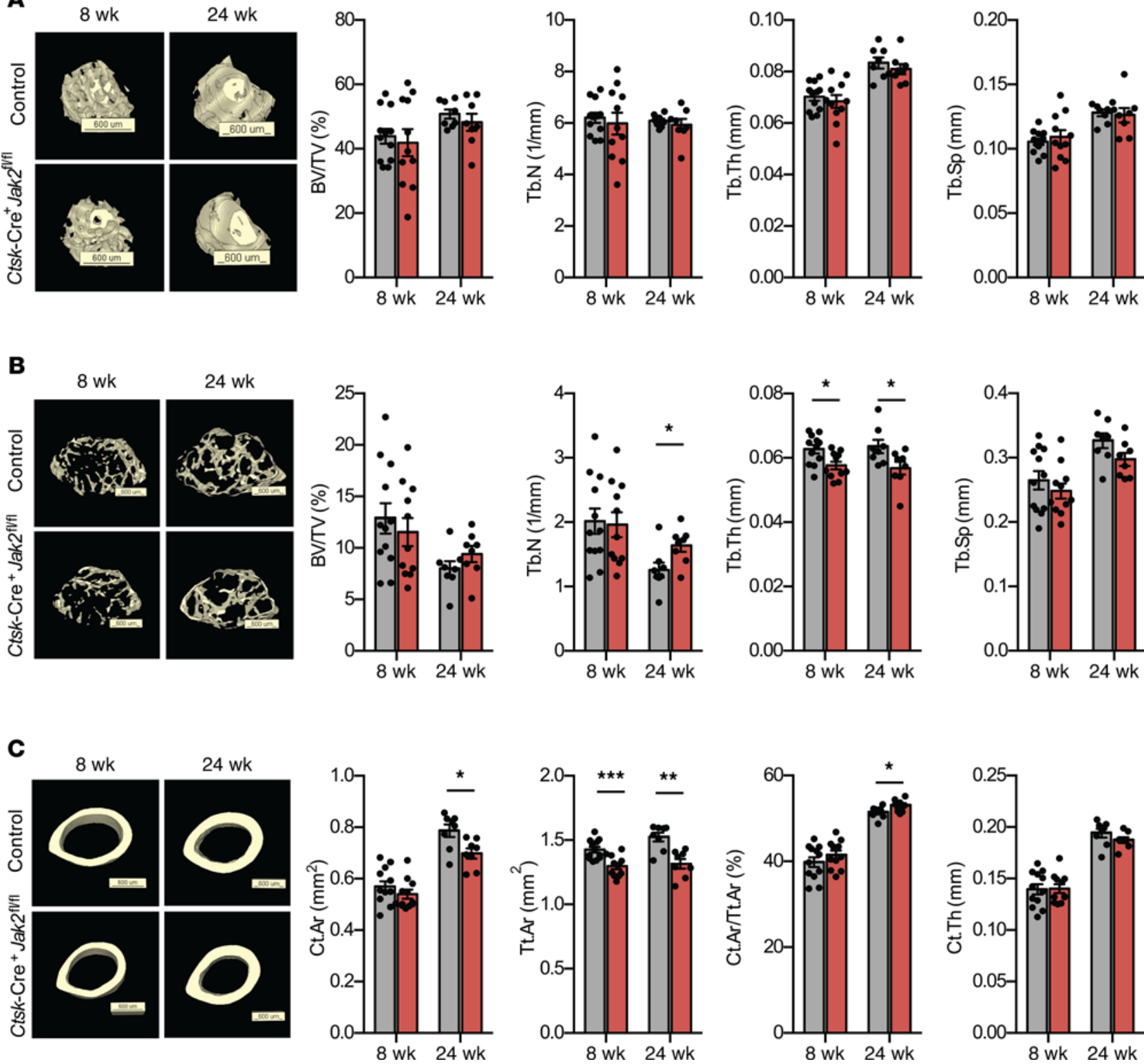

Control

Ctsk-Cre ${ }^{+}$Jak2 $2^{\text {ivf }}$

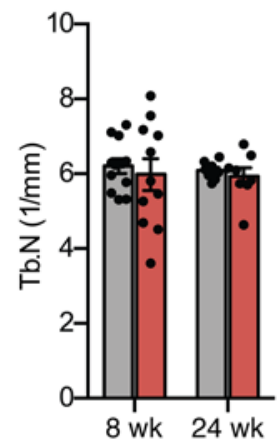

8 wk 24 wk
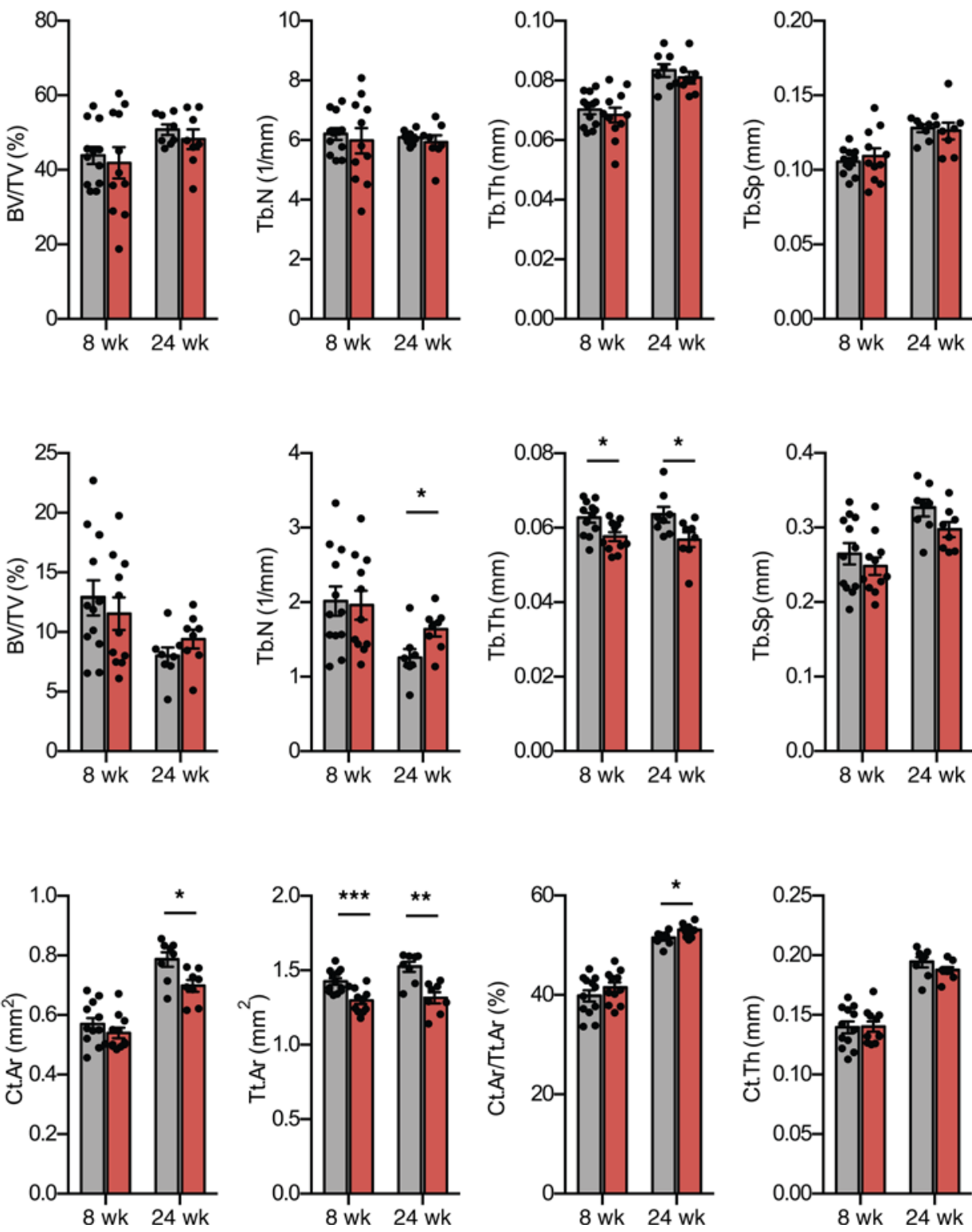

Figure 4. $\mu \mathrm{CT}$ analysis of femurs from Ctsk-Cre+Jak $2^{f / f / f}$ mice. Bone microarchitecture was assessed in female $C t s k$-Cre $J a k 2^{f / f l}$ and control mice at 8 and 24 weeks of age by ex vivo $\mu \mathrm{CT}$. (A) Three-dimensional reconstruction of the femur neck and quantification of percent bone volume (BV/TV), trabecular number (Tb.N), trabecular thickness (Tb.Th), and trabecular separation (Tb.Sp) in control $\left[n=12\right.$ (8 weeks), $n=8$ (24 weeks)] and Ctsk-Cre+ $/ a k 2^{f / f}[n=11$ (8 weeks), $n=8$ (24 weeks)] mice. (B) Three dimensional reconstruction of the distal femur and quantification of BV/TV, Tb.N, Tb.Th, and Tb.Sp in control [ $n$ $=12$ (8 weeks), $n=8$ (24 weeks)] and Ctsk-Cre+Jak $2^{f / f}[n=11$ (8 weeks), $n=8$ (24 weeks)] mice. (C) Three-dimensional reconstruction of the femur midpoint and quantification of cortical area (Ct.Ar), total area (Tt.Ar), percent cortical area to total area (Ct.Ar/Tt. Ar), and cortical thickness (Ct.Th) in control [ $n=12$ (8 weeks), $n=8$ (24 weeks)] and Ctsk-Cre+ Jak $2^{f / f l}[n=11$ ( 8 weeks), $n=8$ (24 weeks)] mice. Data represent mean \pm SEM. Differences between groups were analyzed for statistical significance by 2 -way ANOVA; ${ }^{*} P<0.05,{ }^{* *} P<0.001$.

(Figure 7B). To determine if the decreased expression of IGF1 in bone was specifically from osteoclasts, we first performed IHC for IGF1 in femur sections (Figure 7C). In both control and KO mice, there was diffuse staining for IGF1 in BM cells and chondrocytes, as previously reported (20). In TRAP ${ }^{+}$ multinucleated giant cells, immunostaining for IGF1 was of lower intensity in Ctsk-Cre $\mathrm{Jak}^{f / f l}$ mice compared with controls, while the surrounding BM cells were unaffected. To further confirm a reduction of osteoclast-specific IGF1, we measured Igf1 gene expression in osteoclasts isolated by FACS. We used control and Ctsk-Cre $\mathrm{Jak}^{\text {fl/fl}}$ mice bred to the $m \mathrm{Tm} G$ reporter mouse and isolated the $\mathrm{GFP}^{+}$bone cells. Compared with $\mathrm{GFP}^{-}$cells, purified $\mathrm{GFP}^{+}$cells had a marked increase in expression of Ctsk (Figure 7D) 

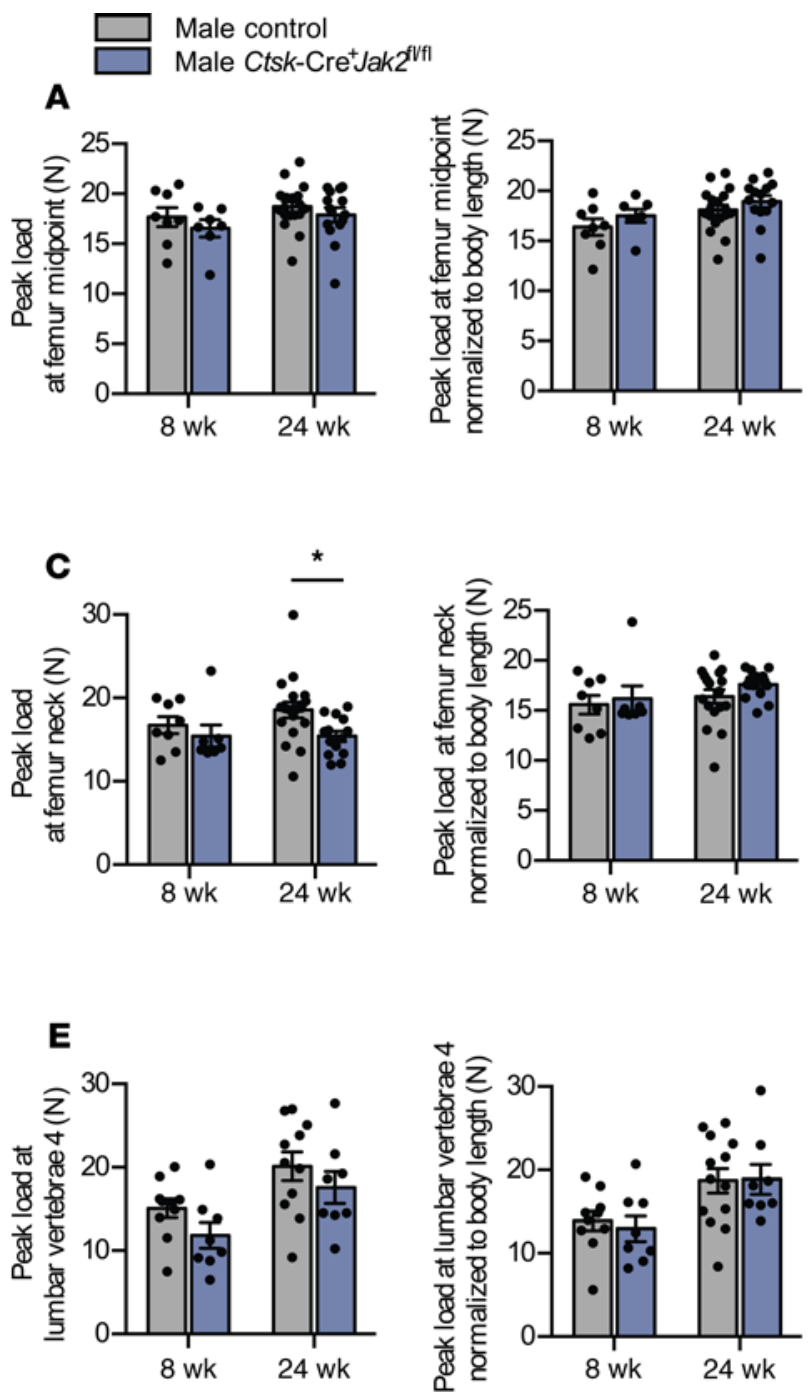
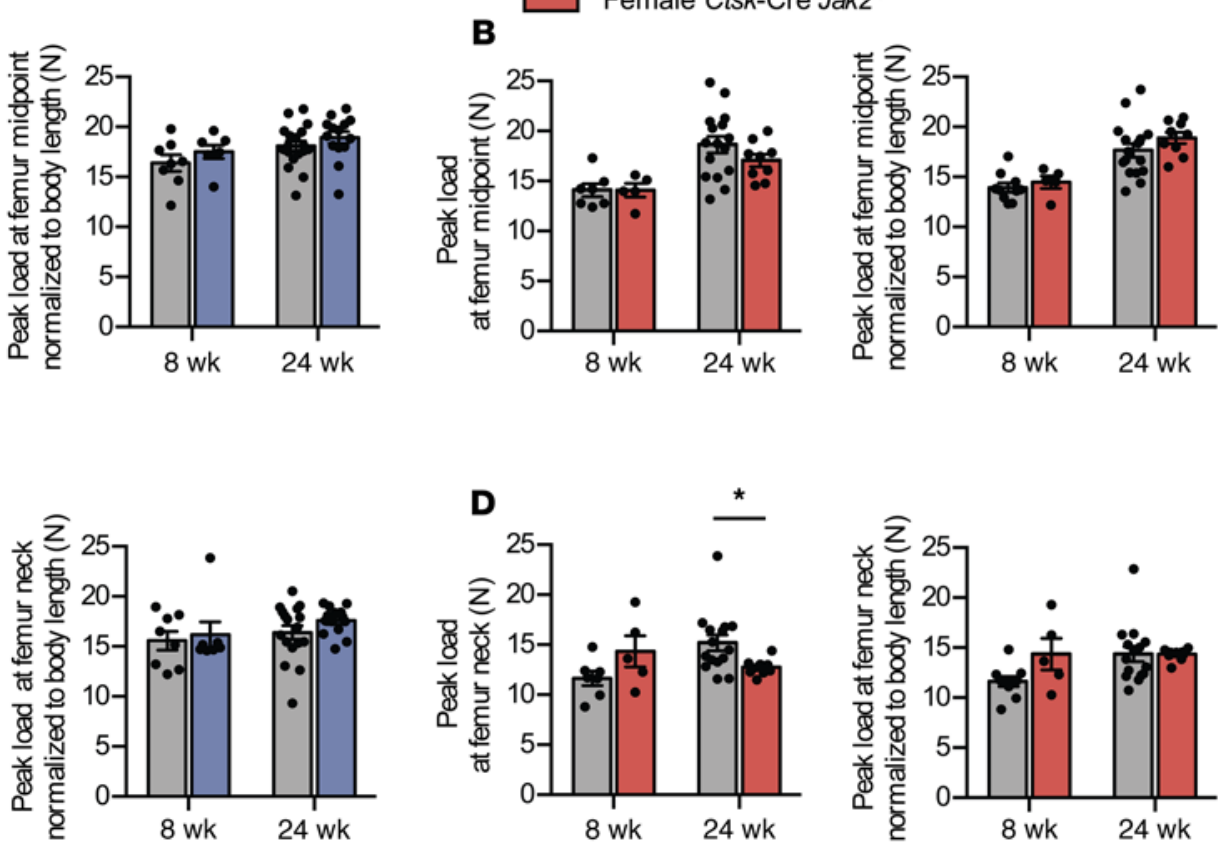

Female control
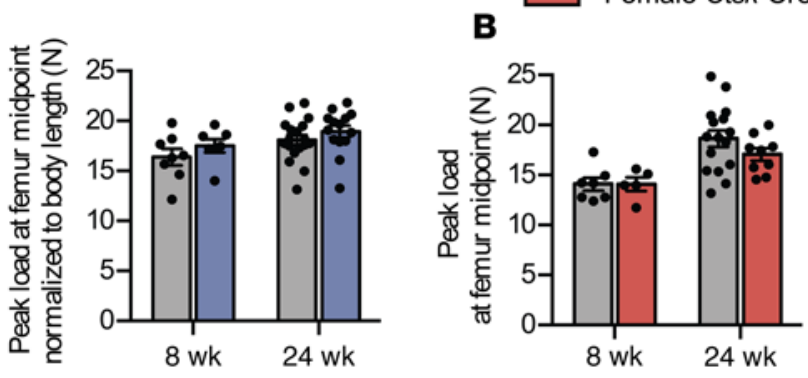

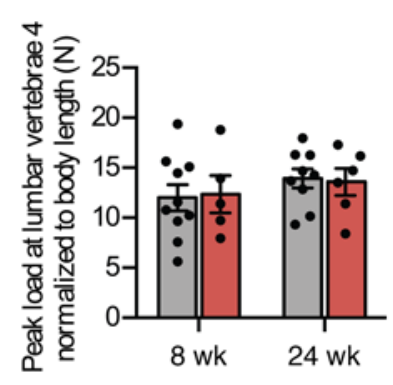

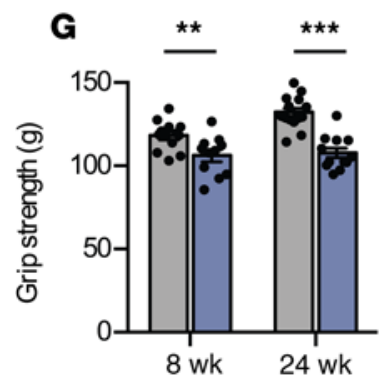
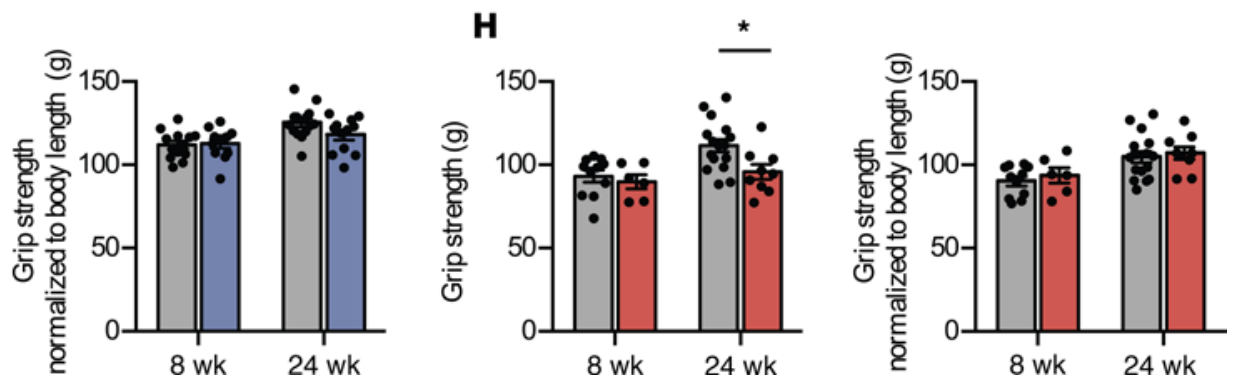

Figure 5. Ctsk-Cre+Jak2 $2^{f / f l}$ mice have proportional reductions in musculoskeletal strength. Musculoskeletal strength of $\left[t s k-C \mathrm{re}^{+}\right] a k 2^{f / f l}$ and control mice was assessed using a materials testing system and grip strength meter. (A) Femur midpoint peak load (left panel) and peak load normalized to body length (right panel) in male control [ $n=8$ ( 8 weeks), $n=18$ (24 weeks)] and Ctsk-Cre+Jak $2^{f / f l}[n=7$ (8 weeks), $n=14$ (24 weeks)] mice. (B) Femur midpoint peak load (left panel) and peak load normalized to body length (right panel) in female control $\left[n=7\right.$ (8 weeks), $n=16$ (24 weeks)] and Ctsk-Cre+Jak $2^{f / f l}[n=5$ (8 weeks), $n=9$ (24 weeks)] mice. (C) Femur neck peak load (left panel) and peak load normalized to body length (right panel) in male control [ $n=8$ ( 8 weeks), $n=18$ (24 weeks)] and Ctsk-Cre+Jak2f/ffl $[n=7$ (8 weeks), $n=14$ (24 weeks)] mice. (D) Femur neck peak load (left panel) and peak load normalized to body length (right panel) in female control $\left[n=7\right.$ ( 8 weeks), $n=15$ (24 weeks)] and Ctsk-Cre+Jak $2^{f / f f}[n=5$ (8 weeks), $n=9$ (24 weeks)] mice. (E) Lumbar vertebra 4 (LV4) peak load (left panel) and peak load normalized to body length (right panel) in male control $\left[n=10\right.$ (8 weeks), $n=11$ (24 weeks)] and Ctsk-Cre ${ }^{+} J a k 2^{f / f f}[n=8$ ( 8 weeks), $n=$ 8 (24 weeks)] mice. (F) LV4 peak load (left panel) and peak load normalized to body length (right panel) in female control [ $n=7$ (8 weeks), $n=9$ (24 weeks)] and Ctsk-Cre ${ }^{+} J a k 2^{f / f l f}[n=5$ ( 8 weeks), $n=6$ (24 weeks)] mice. (C) Grip strength (left panel) and grip strength normalized to body length (right panel) in male control $\left[n=13\right.$ (8 weeks), $n=14$ (24 weeks)] and Ctsk-Cre+Jak2 $2^{f / f l}[n=11$ (8 weeks), $n=12$ (24 weeks)] mice. (H) Grip strength (left panel) and grip strength normalized to body length (right panel) in female control $\left[n=11\right.$ (8 weeks), $n=16$ (24 weeks)] and Ctsk-Cre $J a k 2^{f / f l f}[n=6$ (8 weeks), $n=9$ (24 weeks)] mice. Data represent mean \pm SEM. Differences between groups were analyzed for statistical significance by 2 -way ANOVA; ${ }^{*} P<0.05,{ }^{* *} P<0.01,{ }^{* * *} P<0.001$. 
A
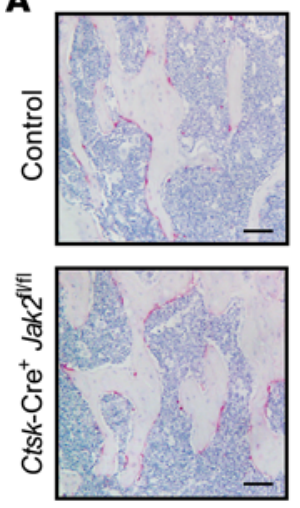

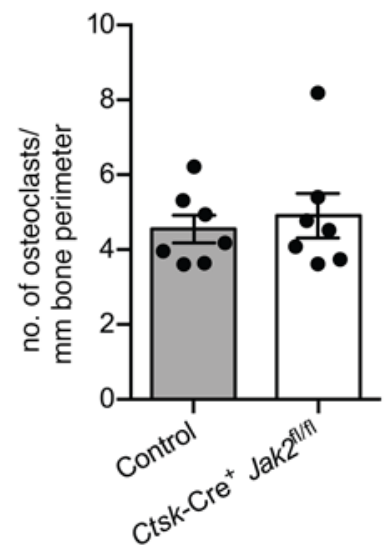

B $\square$ Ctsk-Cre ${ }^{+}$Jak $^{\text {2Vil }}$

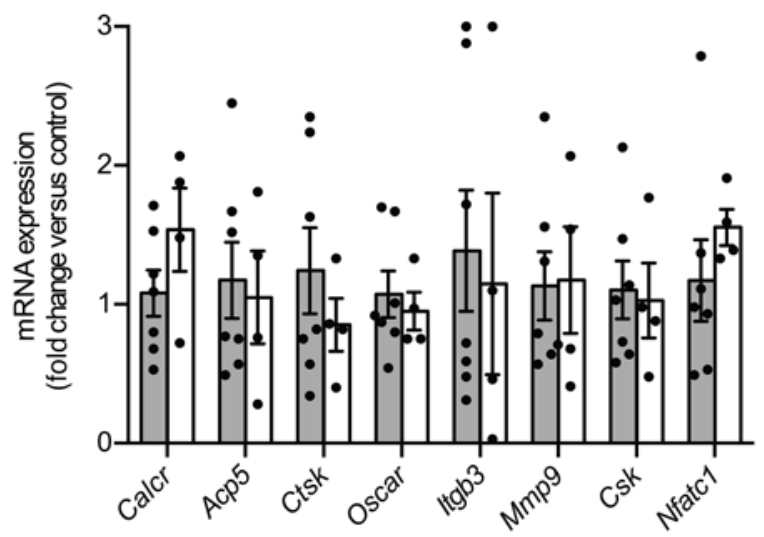

C

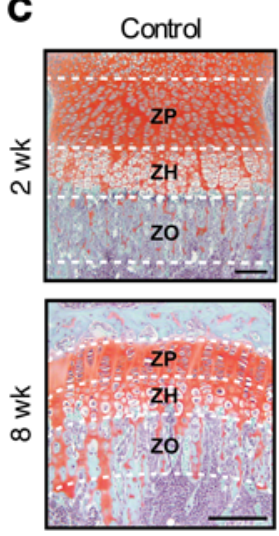

Ctsk-Cre+ Jak $2^{\text {ivil }}$
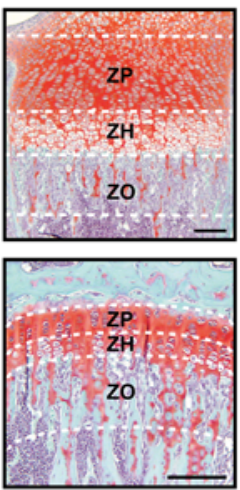
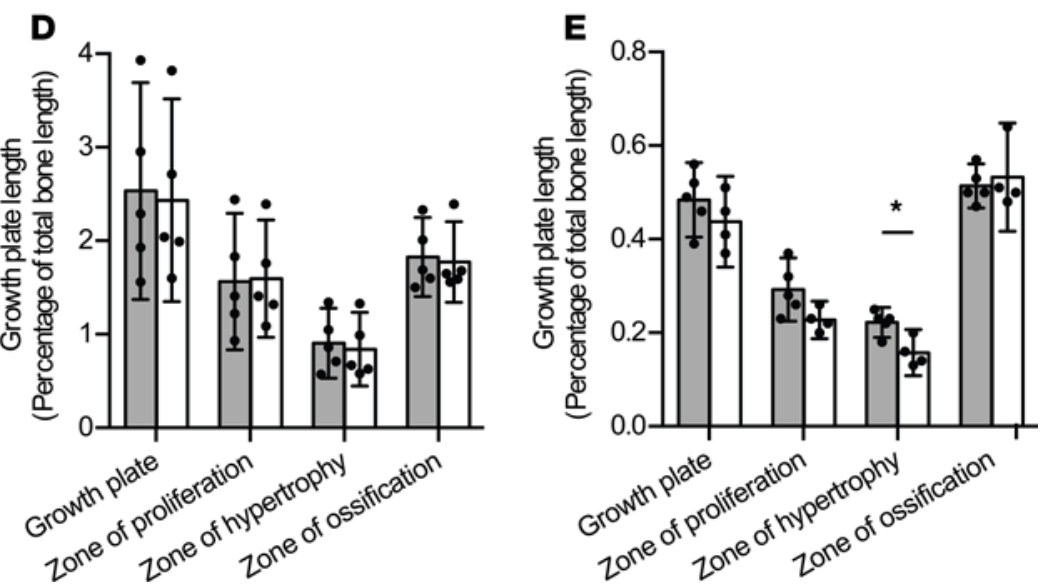

Figure 6. Reduced size of the hypertrophic growth plate zone in Ctsk-Cre $+\mathbf{a k} \mathbf{2}^{\text {fl/f }}$ mice. (A) Representative images of tartrate-resistant alkaline phosphatasestained (TRAP-stained) sections of distal femurs (left) and quantification of osteoclast number per millimeter of bone perimeter (right) in control ( $n=7)$ and Ctsk-Cre+Jak $2^{f / f l}$ mice $(n=7)$. Scale bar: $50 \mu \mathrm{m}$. (B) mRNA expression of calcitonin receptor (Calcr), tartrate-resistant acid phosphatase type 5 (Acp5), cathepsin- $\mathrm{k}$ (Ctsk), osteoclast-associated receptor (Oscar), integrin $\beta 3$ (Itgb3), matrix metalloproteinase 9 (Mmp9), C-terminal Src kinase (Csk), and nuclear factor of activated t cells 1 (Nfatc1) in bone from control $(n=7)$ and Ctsk-Cre $J a k 2^{f / f l}$ mice $(n=4)$. Values are normalized to 185 mRNA levels and presented as fold change over control group. (C) Representative images of Safranin 0-stained sections of tibia growth plates from 2- and 8-week-old control $[n=5$ ( 2 weeks), $n=5$ (8 weeks)] and Ctsk-Cre+Jak $2^{\text {flff }}[n=5$ (2 weeks), $n=4$ (8 weeks)] mice. ZP, zone of proliferation; ZH, zone of hypertrophy; ZO, zone of ossification; growth plate = ZP $+\mathrm{ZH}$ ). Scale bars: $100 \mu \mathrm{m}$. (D) Quantification of tibia growth plate length relative to total bone length in 2-week-old male and female control $(n=5)$ and Ctsk-Cre+ $J a k 2^{f / f l}(n=5)$ mice. (E) Quantification of tibia growth plate length relative to total bone length in 8-week-old male and female control $(n=5)$ and $C t s k$-Cre $J a k 2^{f / f l}(n=4)$ mice. Data represent mean \pm SEM. Differences between groups were analyzed for statistical significance by Student's unpaired $t$ test; ${ }^{*} P<0.05$.

and $A c p 5$ (Figure 7E), consistent with these being mature osteoclasts. Jak2 mRNA was reduced by $62 \%$ in $\mathrm{GFP}^{+}$cells in $\mathrm{Ctsk}$-Cre $J a k 2^{f / /} m \mathrm{Tm} \mathrm{G}^{+}$mice compared with osteoclast Jak2 heterozygous (Ctsk-Cre $\mathrm{Jak}^{2 / /+}$ $m T m G^{+}$) control mice (Figure $7 \mathrm{~F}$ ). Similarly, Igf1 mRNA was reduced by approximately $70 \%$ in $\mathrm{GFP}^{+}$ cells from Ctsk-Cre $\mathrm{Jak}^{\ell / f} m \mathrm{mTm} \mathrm{G}^{+}$mice compared with osteoclast Jak2 heterozygous mice (Figure 7G). No difference in either Jak2 or Igf1 expression was detected in GFP- cells. Overall, these findings are consistent with reduced osteoclast-specific IGF1 expression in $\mathrm{Ctsk}_{\mathrm{C}} \mathrm{Cre}^{+} J a k 2^{\text {f/f }}$ mice.

$C t s k-C r e^{+} I g f I^{\text {H/f }}$ mice demonstrate postnatal growth restriction. Given our results showing growth defects with reduced osteoclast-derived IGF1 in $C t s k-\mathrm{Cre}^{+} J a k 2^{l / f}$ mice, we hypothesized that a causal link exists between osteoclast-derived IGF1 and postnatal growth. To test this hypothesis, we generated osteoclast-specific IGF1-KO mice (Ctsk-Cre+ Igff $f^{f / f}$ mice). IHC performed on femur sections confirmed loss of IGF1 specifically in $\mathrm{TRAP}^{+}$multinucleated giant cells in $C t s k-\mathrm{Cre}^{+} I g f f^{1 / f /}$ mice (Figure 8A). Similar to Ctsk-Cre ${ }^{+} J a k 2^{t / f}$ mice, both male and female $C t s k-\mathrm{Cre}^{+} \mathrm{Igff^{ \text {I/f } }}$ mice had small but statistically significant reductions in body weight (Figure 8, B and C), body length (Figure 8, D and E), and femur length (Figure 8, F and G) compared with littermate controls at 8 weeks of age, but not at 2 weeks. The extent of reduction in growth parameters was 
A

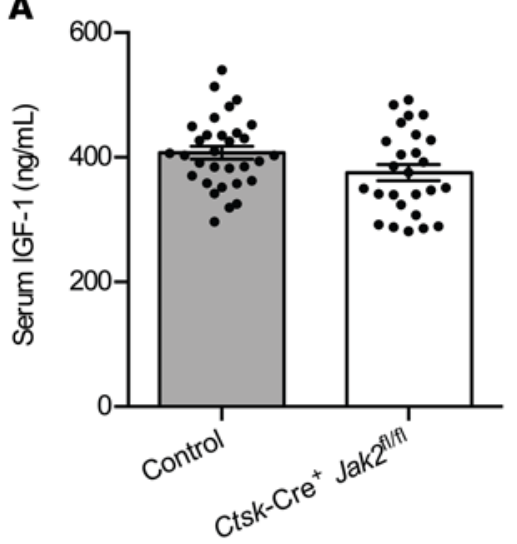

C
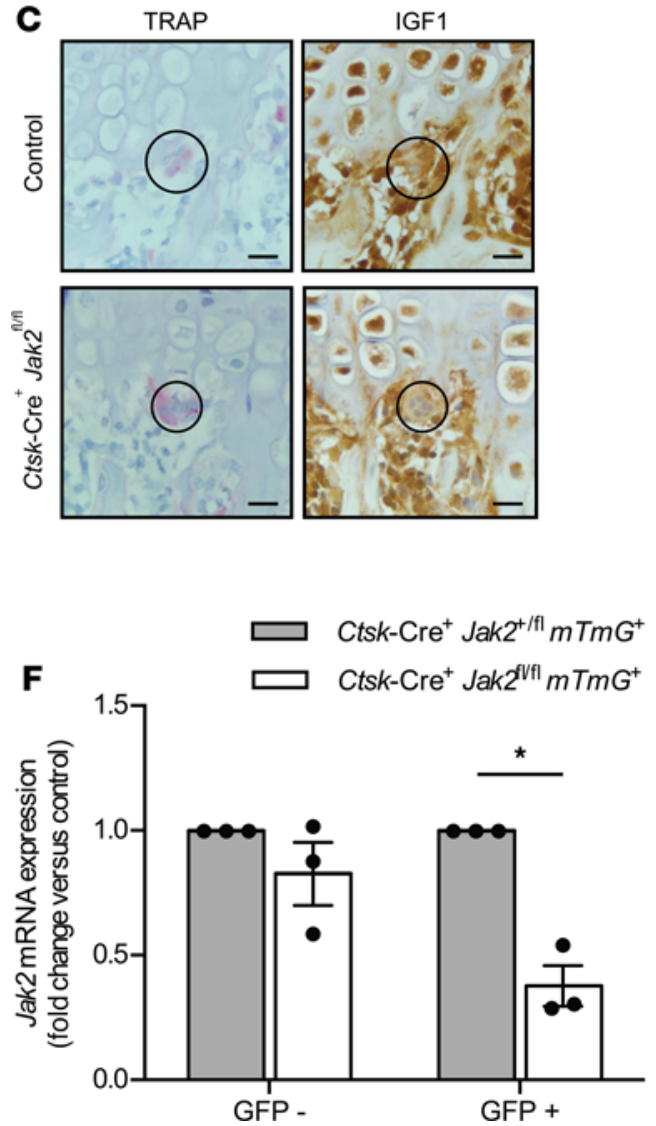

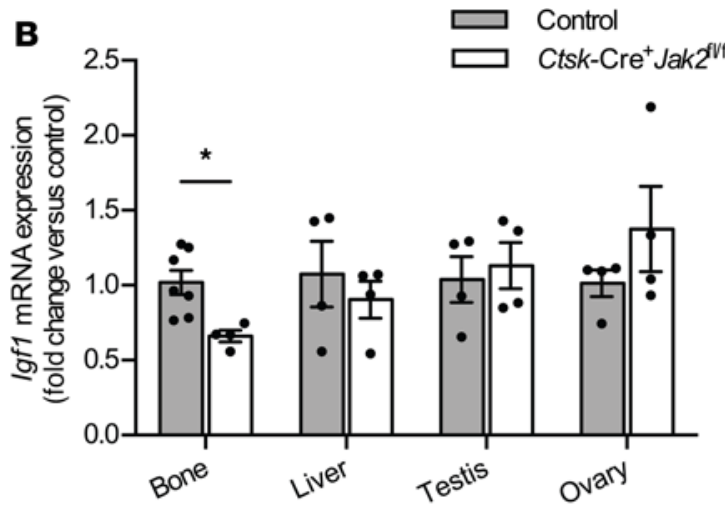

D

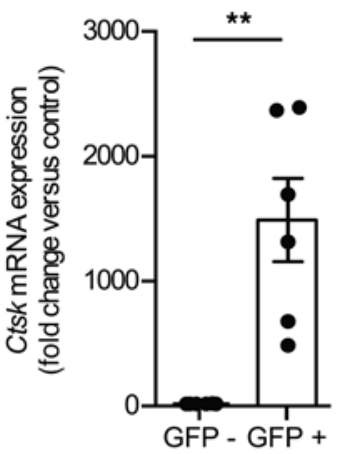

E

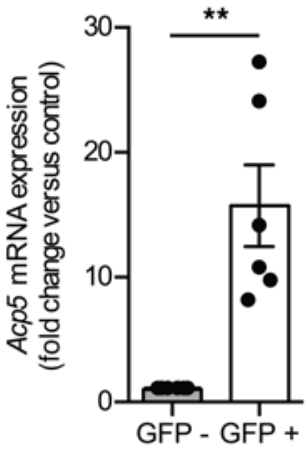

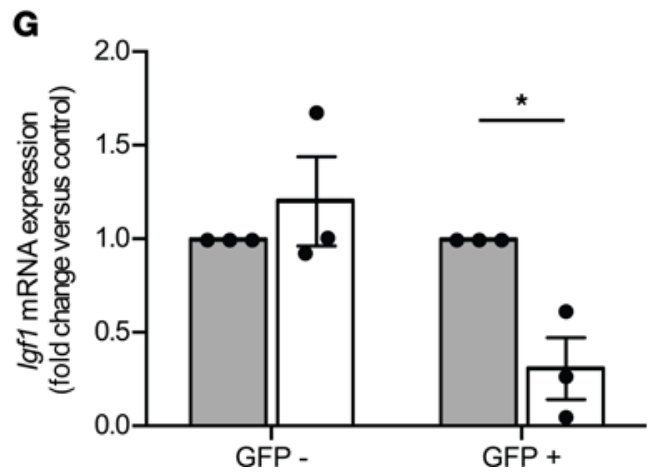

Figure 7. Reduced osteoclast-specific expression of IGF1 in Ctsk-Cre+Jak $2^{f / f f}$ mice. Expression of ICF1 was assessed in tissues and osteoclasts from 8-week-old Ctsk-Cre+Jak $2^{f / f l}$ and control mice. (A) Serum IGF1 concentrations in male and female control $(n=31)$ and Ctsk-Cre $J a k 2^{f / f l}(n=26)$ mice at 8 weeks of age. (B) mRNA expression of $I g f 1$ in bone (distal femur and proximal tibia), liver, testis, and ovary from control $\left(n=4,\left[n=7\right.\right.$ for bone]) and Ctsk-Cre $J a k 2^{f / f l}$ mice $(n=4)$ mice. Values are normalized to 185 mRNA levels and presented as fold change over control group. (C) Representative images of femur from control ( $n=3$ ) and $C t s k$-Cre ${ }^{+}$ Jak $2^{f / f l}$ mice $(n=3)$ stained for TRAP and immunostained for IGF1. Staining was performed on adjacent serial sections. The circles highlight TRAP+ multinucleated

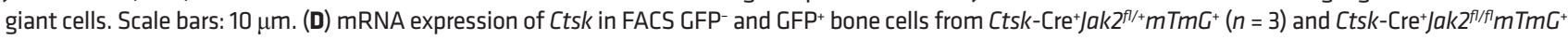
mice $(n=3)$. Values are normalized to 185 mRNA levels and presented as fold change over GFP- cells. (E) mRNA expression of Acp5 in FACS GFP- and GFP+ bone

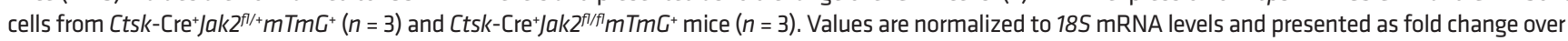

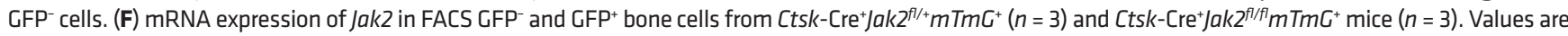
normalized to 185 mRNA levels and presented as fold change over control group. (C) mRNA expression of lgf1 in FACS GFP- and GFP+ bone cells from Ctsk-Cre ${ }^{+}$

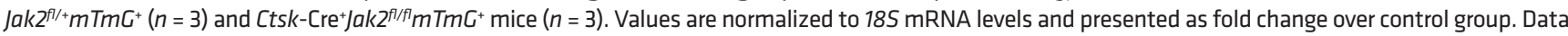
represent mean \pm SEM. Differences between groups were analyzed for statistical significance by Student's unpaired $t$ test; ${ }^{*} P<0.05$, ${ }^{* *} P<0.01$. 
A
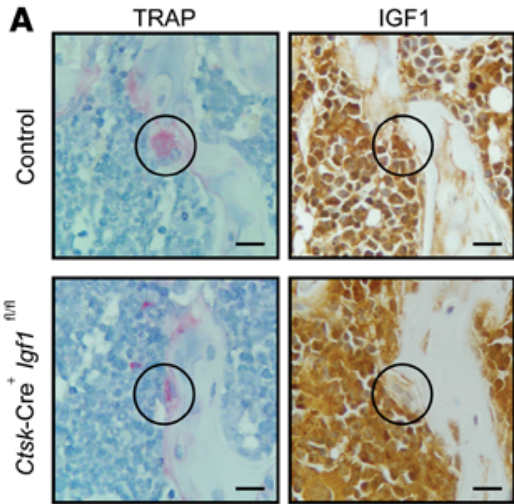

B
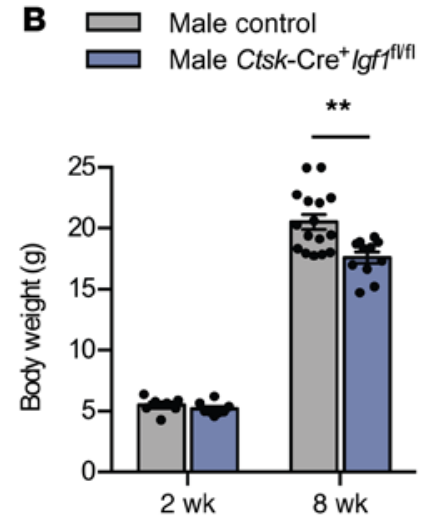

C

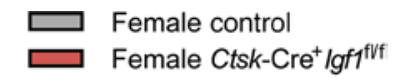

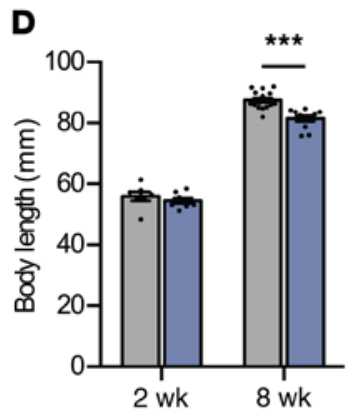
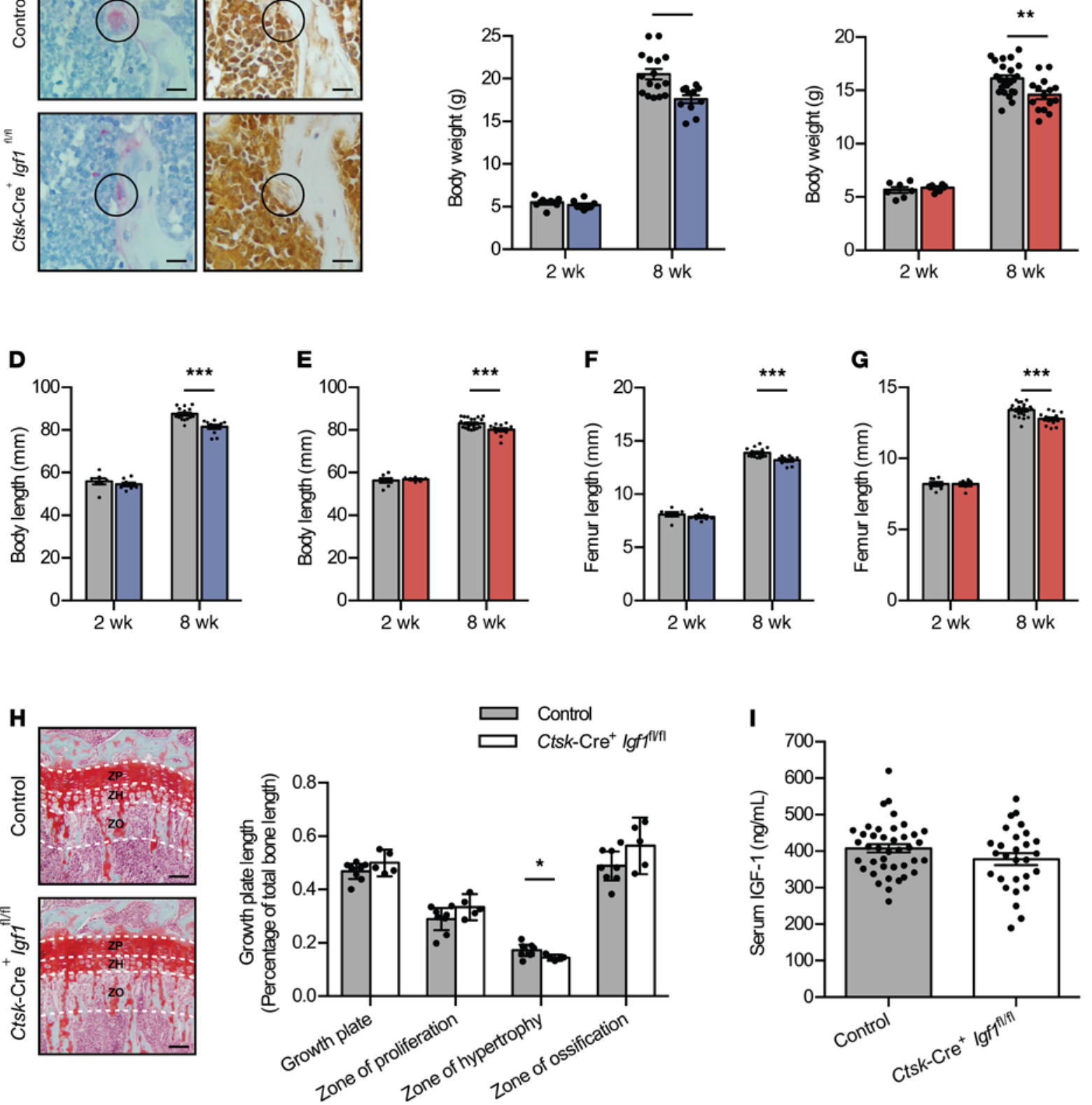

Figure 8. Ctsk-Cre+lgf $f^{f / f l}$ mice demonstrate postnatal growth restriction. Ctsk-Cre+lgf $f^{f / f l}$ and littermate control mice were followed until 8 weeks of age, and growth parameters were assessed. (A) Representative images of femur from control $(n=3)$ and Ctsk-Cre ${ }^{+} / g f f^{f / f l}$ mice $(n=3)$ stained for TRAP and immunostained for IGF1. Staining was performed on adjacent serial sections. The circles highlight TRAP ${ }^{+}$multinucleated giant cells. Scale bars: $10 \mu \mathrm{m}$. (B) Body weight of male control and Ctsk-Cre+lgf fl/fl mice. (C) Body weight of female control and Ctsk-Cre+lgff/lfl mice. (D) Body length of male control and

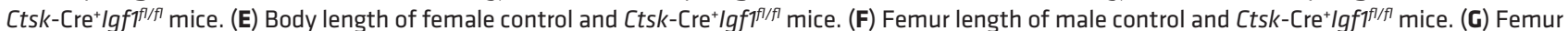
length of female control and Ctsk-Cre ${ }^{+} / g f f^{f / f l}$ mice. Control males $\left[n=7\right.$ (2 weeks), $n=16$ (8 weeks)] and Ctsk-Cre $/ g f 1^{f / f l}$ males $[n=8(2$ weeks), $n=11(8$ weeks)] mice. Control females [ $n=7$ (2 weeks), $n=23$ (8 weeks)] and Ctsk-Cre+lgf $1^{f / f l}$ females $[n=6$ ( 2 weeks), $n=14$ (8 weeks)]. (H) Representative photographs of Safranin 0-stained tibia growth plates and quantification of tibia growth plate length relative to total bone length in 8 -week-old control $(n=8)$ and Ctsk-Cre ${ }^{+} / g f f^{f / f / P}(n=5)$ mice (ZP, zone of proliferation; $\mathrm{ZH}$, zone of hypertrophy; ZO, zone of ossification; growth plate $\left.=\mathrm{ZP}+\mathrm{ZH}\right)$. Scale bars: $100 \mu \mathrm{m}$. (I) Serum IGF1 concentrations in male and female control $(n=40)$ and Ctsk-Cre $\lg f f^{f / f l}(n=28)$ mice at 8 weeks of age. Data represent mean \pm SEM. Differences between groups were analyzed for statistical significance by 2-way ANOVA or Student's unpaired $t$ test; ${ }^{*} P<0.05,{ }^{* *} P<0.01,{ }^{* *} P<0.001$.

also similar between the 2 gene $\mathrm{KO}$ models. Additionally, histological analysis of the growth plate showed a decrease in the size of the $\mathrm{ZH}$ without changes in the $\mathrm{ZO}$ (Figure $8 \mathrm{H}$ ). Serum IGF1 concentrations were similar in control and Ctsk-Cre $I g f 1^{f l f l}$ mice (Figure 8I). Overall, these findings parallel those of Ctsk-Cre $J a k 2^{f / f l}$ mice and support the hypothesis that osteoclast-derived IGF1 regulates postnatal growth. 
A
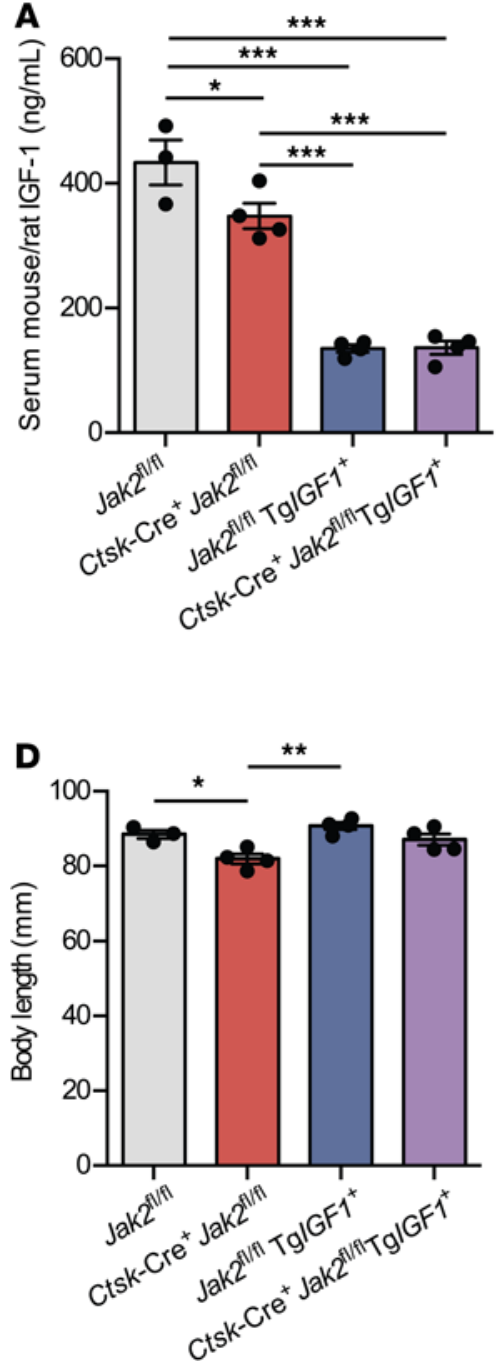

B
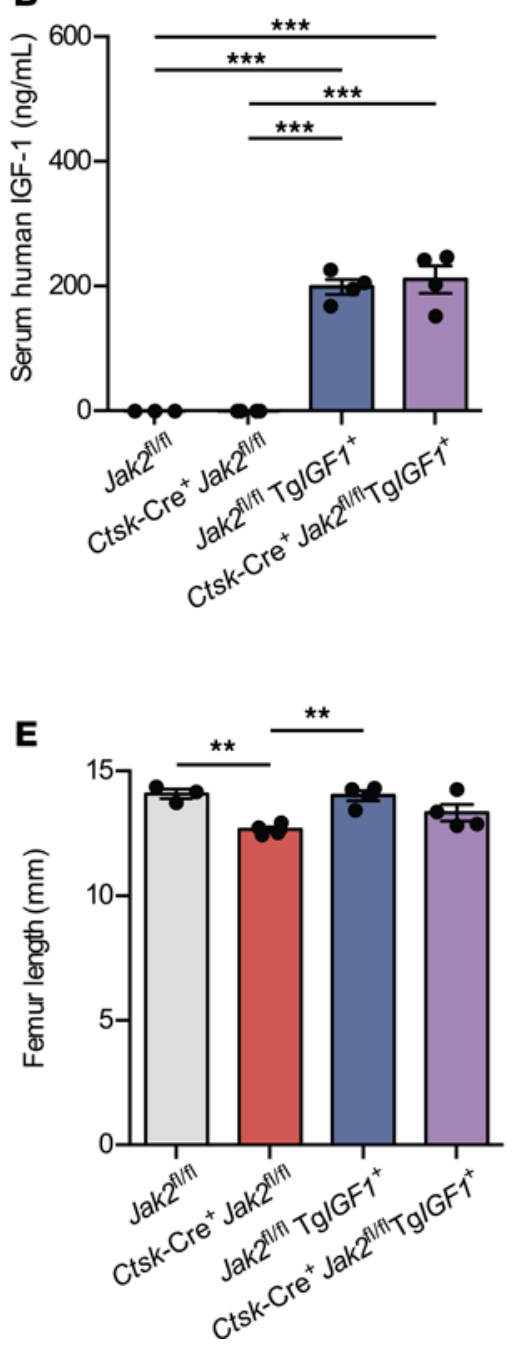

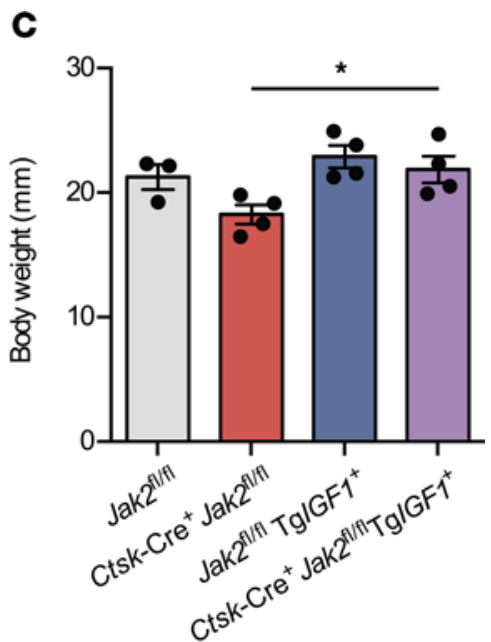

Figure 9. Transgenic overexpression of IGF1 rescues growth defects in Ctsk-Cre+Jak $2^{f / f l}$ mice. Ctsk-Cre ${ }^{+}$Jak $2^{\text {fl/fl }}$ mice were bred to a transgenic mouse overexpressing human IGF1 (Tg/GF $1^{+}$), and growth parameters of male offspring were assessed at 8 weeks of age. (A-E) Serum mouse/ rat IGF1 concentrations (A), serum human IGF1 concentrations (B), body weight (C), body length (D), and femur length $(\mathbf{E})$ of Jak $2^{f / / f}(n=3)$, Ctsk-Cre+Jak2 $2^{f / f l}(n=4)$, Jak2 $2^{f / f f} \operatorname{Tg} / G F 1^{+}(n=4)$, and

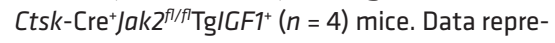
sent mean \pm SEM. Differences between groups were analyzed for statistical significance by 1-way ANOVA; ${ }^{*} P<0.05,{ }^{* *} P<0.01,{ }^{* * *} P<0.001$.

Transgenic overexpression of IGF1 rescues growth defects in Ctsk-Cre $\mathrm{Jak}^{\mathrm{flfl}}$ mice. Since osteoclast expression of IGF1 was found to play a role in body size determination, we sought to determine if the decreased expression of IGF1 in Ctsk-Cre $J a k 2^{f l f l}$ mice had a causal role in their attenuated growth. To this end, we used a transgenic IGF1-overexpressing mouse, which expresses human IGF1 ([h]IGF1) under control of the transthyretin (Ttr) promotor to increase circulating IGF1. This model has previously been shown to overcome both endocrine and local deficits in IGF1 in order to restore normal growth (20). Analysis of serum showed equivalent reductions in circulating mouse IGF1 in both control and $\mathrm{Ctsk}_{\mathrm{Cre}} \mathrm{Cak}^{\mathrm{fl} / \mathrm{fl}}$ mice carrying the (h)IGF1 transgene (Figure 9A). Moreover, levels of human IGF1 in circulation were also similar between these mice (Figure 9B). Mouse IGF1 was likely reduced in the presence of the (h)IGF1 transgene due to feedback inhibition of endogenous IGF1. (h)IGF1 expression did not alter growth parameters in control mice. This is consistent with previous studies showing no effect on body length and femur length in (h)IGF1-expressing mice (21). In Ctsk-Cre $\mathrm{Cak}^{2 / / f l}$ mice, however, transgenic expression of (h)IGF1 resulted in normalization of body weight (Figure 9C), body length (Figure 9D), and femur length (Figure 9E) at 8 weeks of age. These findings suggest a causal role between reduced IGF1 expression in Ctsk-Cre ${ }^{+} J a k 2^{f l f l}$ mice and postnatal growth restriction.

\section{Discussion}

In this study, we investigated the in vivo role of JAK2 in osteoclasts using Cre/Lox-P-mediated gene deletion. Although Oc-JAK2-KO mice demonstrated postnatal growth restriction, we did not identify any structural or functional alterations in bone, and any changes observed were proportional to the change in body size, suggesting that JAK2 is not essential for canonical bone resorptive function in osteoclasts. We assessed 
multiple measures of bone homeostasis in both sexes and at multiple time points. Since peak trabecular bone mass is achieved by 8 weeks and then declines (22), our data show that osteoclast JAK2 is dispensable for both normal bone development and in modulating age-related bone loss.

Previous studies have hinted at a potential role for JAK2 in osteoclast biology, since cytokines and growth factors that signal through JAK2 have been reported to modulate osteoclastogenesis (23). For example, growth hormone has been reported to promote osteoclastogenesis from unfractionated bone cells through direct effects on osteoclasts and indirectly through effects on stromal cells (24). IL-6 has been shown to inhibit osteoclastogenesis in BM macrophages and RAW 264.6 cell, which is dependent on STAT3 activation (25). IL-12, which also signals through JAK2-mediated STAT phosphorylation, inhibits osteoclastogenesis indirectly through induction of IFN- $\gamma$ in stromal and immune cells (26). RANKL, which is essential for osteoclast formation, is also dependent on JAK-STAT signaling (27). RANKL-stimulated osteoclast formation is associated with reduced protein expression of JAK1, which prevents IFN- $\beta$ inhibition of osteoclast lineage commitment. However, there are no alterations in JAK2 or JAK3 levels upon RANKL stimulation, suggesting that JAK2 is not essential, whereas JAK1 may be essential in the differentiation of osteoclasts. In terms of studies investigating JAK2 specifically, the JAK2 inhibitor AG490 was shown to promote osteoclast survival in vitro upon RANKL withdrawal (11). There is, however, doubt about the specificity of AG490 for JAK2, since it has been reported to also inhibit JAK3, STAT1/3/5, activating protein 1, and mitogen-activated protein kinase pathways; thus, it is unclear whether the effects observed in this study can be attributed specifically to inhibition of JAK2 (28). Overall, the studies linking JAK2-mediated signaling with osteoclast differentiation and survival are limited by the fact that osteoclast biology has only been investigated in vitro and that many of the effects seen may be due to indirect effects on nonosteoclast cells. Based on the results from our current study, we find that JAK2 does not have an appreciable effect on osteoclast formation or resorptive function in vivo. Further investigation, however, may be needed to determine if there is a role of osteoclast JAK2 in the setting of advanced age or sex hormone deficiency.

Despite the lack of bone resorptive effects seen in this study, Oc-JAK2-KO mice displayed significant postnatal growth restriction. Many in vivo gene $\mathrm{KO}$ studies have been performed in osteoclasts resulting in growth defects, which often arise in conjunction with impaired osteoclast function in vivo resulting in osteopetrosis (14-15). This is accompanied by an increase in bone mass and other defects, including doming of the skull, delayed tooth eruption, and radio densities within regions of the BM. In our study, Oc-JAK2-KO mice did not display an increase in bone mass or any stigmata of osteopetrosis; therefore, it is unlikely that growth restriction occurred as a result of impairment in the traditional osteoclast function in bone resorption. We therefore propose a potentially novel role for osteoclasts in determining body growth independently of their canonical function in bone mineral homeostasis. Since JAK2 is a well-established regulator of IGF1 expression, most classically from the liver (16-19), we hypothesized that JAK2 signaling in osteoclasts might similarly regulate IGF1 expression. The body growth restriction observed in Oc-JAK2-KO mice was associated with reduced expression of IGF1 in bone and osteoclasts. Overexpression of IGF1 abolished these growth defects, suggesting a causal role between JAK2-mediated IGF1 expression in osteoclasts and determination of body size. Additionally, when IGF1 was directly targeted in osteoclasts, this produced almost identical phenotypic findings compared with Oc-JAK2-KO mice. Together, these data support a role for osteoclast-derived IGF1 as a small but significant contributor to postnatal growth.

IGF1 is a ubiquitously expressed hormone and plays an essential role in postnatal growth (29). It is well established that approximately $75 \%$ of circulating IGF1 is derived from the liver (30-31). Our understanding, however, of extrahepatic sources of IGF1 remains unclear, and there is an emerging recognition of the importance of autocrine and paracrine functions of IGF1. For example, targeted deletion of IGF1 in chondrocytes (32), osteoblasts (33), or osteocytes (34) produces unique effects on bone homeostasis and growth, without affecting circulating IGF concentrations. Our study provides evidence that IGF1 in osteoclasts similarly plays an important role in postnatal growth. Given that we saw changes in IGF1 in osteoclasts and bone, but not in circulating IGF1 concentrations, this would support a role for local autocrine or paracrine actions of osteoclast-derived IGF1. It has previously been shown that IGF1 is an important stimulator of chondrocyte hypertrophy (35), and in our study, a reduction in the hypertrophic zone was the only histological change seen in the growth plate. Thus, it is reasonable to suspect that osteoclast-derived IGF1 might act locally on chondrocytes in this zone, since they are located immediately adjacent to the $\mathrm{ZO}$, where numerous active osteoclasts are located. We found the $\mathrm{ZH}$ to be reduced in size without associated changes in the overall size of the growth plate. This may be due to inadequate sample size or missing growth plate changes at other critical time 
points during this dynamic process. Overall, further research is needed to delineate the specific local actions of osteoclast-derived IGF1; however, the details of this are beyond the scope of our current study.

In conclusion, this study describes a potentially novel role for Oc-JAK2 and IGF1 in the regulation of postnatal growth and determination of body size. Apart from the role of Oc-JAK2 in body growth, we show that under physiologic conditions, Oc-JAK2 appears to be dispensable for the canonical function of osteoclasts and not essential to the development and homeostasis of bone mass, strength, or microarchitecture in vivo.

\section{Methods}

Animals. Ctsk-Cre mice (Ctsk $k^{\text {tm1[cre]ska }) ~(36) ~ w e r e ~ o b t a i n e d ~ f r o m ~ W e n t i a n ~ Y a n g ~(B r o w n ~ U n i v e r s i t y ~ A l p e r t ~}$

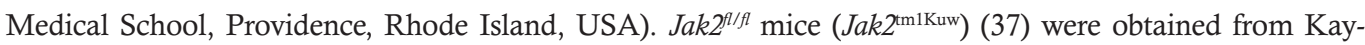
Uwe Wagner (Wayne State University, Detroit, Michigan, USA). $m$ TmG reporter mice (Gt[ROSA]26Sor

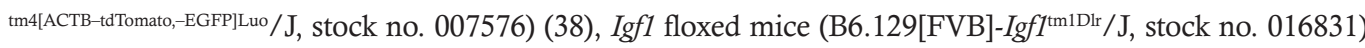
(39), and transgenic IGF1-overexpressing mice (FVB-Tg[Ttr-IGF1]1Sykr/J, stock no. 012662) (21) were obtained from the Jackson Laboratory. Oc-JAK2-KO mice were generated by breeding heterozygous Ctsk-

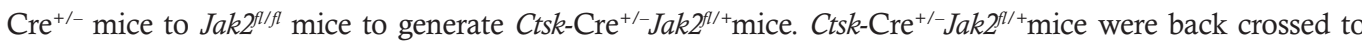
$J a k 2^{f / f l}$ mice to generate Oc-JAK2-KO mice $\left(C t s k-C r e^{+/-} J a k 2^{f / f}\right)$ and littermate control mice (Jak2 $2^{f / f l}$ and Ctsk$\left.\mathrm{Cre}^{+/-} J a k 2^{f /+}\right)$. Both $J a k 2^{f / f l}$ and $\mathrm{Ctsk}-\mathrm{Cre}{ }^{+/-} J a k 2^{f /+}$ controls were used for all experiments unless otherwise specified. There were no phenotypic differences in any outcomes between Jak2 $2^{f / f l}$ and $C t s k-C r e^{+/-} J a k 2^{f /+}$ mice. Osteoclast-specific IGF1-KO mice (Ctsk-Cre $\left.{ }^{+} I f f^{f l / f t}\right)$ were generated using the same breeding scheme. For reporter mouse experiments, $C t s k-\mathrm{Cre}^{+/-}$mice were bred to $m \mathrm{Tm}_{m} \mathrm{H}^{+/-}$mice to generate $\mathrm{Ctsk}-\mathrm{Cre}^{+/-} m \mathrm{TmG}^{+/-}$ mice. For transgenic IGF1 rescue experiments, Ctsk-Cre ${ }^{+/-} J a k 2^{f / f l}$ mice were bred to $J a k 2^{f / f f} \mathrm{Tg} I G F 1^{+/-}$to generate control ( $\left.J a k 2^{f / f f}\right)$, KO (Ctsk-Cre $\left.{ }^{+/-} J a k 2^{f / f f}\right)$, control with IGF1 overexpression ( $\left.J a k 2^{f / / f} \mathrm{Tg} I G F 1^{+/-}\right)$, and $\mathrm{KO}$ with IGF1 overexpression (Ctsk-Cre ${ }^{+/-} J a k 2^{f / f l} \mathrm{TgIGF1^{+/- }}$ ) mice. Mice were housed in a pathogen-free facility with a 12-hour light-dark cycle and free access to water and standard rodent chow (5\% energy from fat; Harlan Teklad). Body weights were measured weekly beginning at 2 weeks of age. Measurements of body length and bone length were taken at the time of sacrifice using a digital calliper.

$D X A$ and $\mu C T$. BMD of excised femurs and LV1-3 was measured by DXA (Orthometrix) using a specialized software program (Host Software version 3.9.4; Scanner Software version 1.2.0, Orthometrix). Calibration of the DXA was performed daily using quality control and quality assurance standards. Femurs and LV1-3 were scanned using a resolution of $0.1 \mathrm{~mm} \times 0.1 \mathrm{~mm}$ and at a speed of $2 \mathrm{~mm} / \mathrm{s}$.

For assessment of bone microarchitecture, left femurs were scanned ex vivo using $\mu \mathrm{CT}$ (Skyscan 1176, Bruker microCT). The bones were wrapped in parafilm to retain moisture during scanning and placed axially in a foam holder for scanning using a $9 \mu \mathrm{m}$ isotropic voxel size, $0.25 \mathrm{~mm}$ aluminum filter, voltage of 45 $\mathrm{kV}$, tube current of $545 \mu \mathrm{A}, 850 \mathrm{~ms}$ exposure time, and 0.2 degree rotation step. Scans were performed over $180^{\circ}$ without using frame averaging. To reconstruct scanned images, Graphics Processing Unit-acceleration (GPU-acceleration) (GPUReconServer, Skyscan, Bruker microCT) and NRecon Reconstruction 64-bit software (Skyscan, Bruker microCT) were used. Except for variable misalignment compensations, all scanned samples within each skeletal site received the same corrections to smoothing, ring artifacts, beam hardening, and defect pixel masking. Reconstructed images were then reoriented (DataViewer software, version 1.5.0, Skyscan, Bruker microCT), and the transaxial images were saved. Regions of interest (ROIs) were selected from transaxial images and saved as new data sets using CTAnalyzer software (Skyscan Bruker microCT). At the femur neck, an ROI of the trabecular region was manually drawn to a few pixels away from the endocortical boundary and consisted of $1.680 \mathrm{~mm}$ (191 slices), starting at the distal edge of the femur head and extending toward the diaphysis of the femur. At the distal femur, the ROI of the trabecular region was manually drawn a few pixels away from the endocortical boundary and consisted of $0.879 \mathrm{~mm}$ (100 slices), starting $0.616 \mathrm{~mm}$ (70 slices) proximal to the metaphyseal edge of the growth plate and extending toward the femur neck. Another ROI was manually drawn around the midpoint region of the femur, rich in cortical bone, and consisted of $0.879 \mathrm{~mm}$ (100 slices) spanning above and below the femur midpoint. Local adaptive thresholding was used to segment the trabecular bone from the background (femur neck: lower threshold = 74 , upper threshold $=255$, radius $=6$, constant $=0$; distal femur: lower threshold $=71$, upper threshold $=$ 255 , radius $=6$, constant $=0$ ) and global thresholding (femur: lower threshold $=124$, upper threshold $=255$ ) for segmenting the cortical bone from the background at the femur midpoint. At the femur neck and distal femur, the trabecular outcomes determined were bone volume fraction (BV/TV), trabecular number (Tb.N), trabecular thickness (Tb.Th), and trabecular separation (Tb.Sp). Cortical bone outcomes determined at the 
femur midpoint were total cross-sectional area inside the periosteal envelope (Tt.Ar), cortical bone area (Ct. Ar), cortical area fraction (Ct.Ar/Tt.Ar), and average cortical thickness (Ct.Th).

Biomechanical strength testing. Bone strength was measured using a Materials Testing System (Model 4442, Instron Corp.) and specialized software (Bluehill 2, Instron Corp.). To assess bone strength at skeletal sites rich in cortical bone, the peak load at the femur midpoint was determined by 3-point bending. To assess skeletal sites rich in trabecular bone, the maximum compressive force at the femur neck and LV4 was measured. Grip strength was measured using a mouse/rat grip strength meter (Model BIO-GS3, Bioseb). Forelimb grip strength was measured according to the manufacturer's instructions. Ten consecutive grip strength tests were performed, and the best 3 attempts were averaged to give maximal grip strength.

Histology. Femurs and tibia were cleaned and fixed in 10\% neutral buffered formalin for $>48$ hours and decalcified in 14\% EDTA ( $\mathrm{pH}$ 7.2) for 2 weeks, and they were then paraffin embedded and cut into $5 \mu \mathrm{m}$ sagittal sections. For TRAP staining, rehydrated sections were incubated in TRAP staining solution (sodium acetate $[0.11 \mathrm{M}]$, L-tartaric acid [76.0 mM], napthol AS-MX phosphate [0.27M], ethylene glycol monoethyl ether $[0.5 \% \mathrm{v} / \mathrm{v}]$, and Fast Red Violet LB Salt [1.59M]; $\mathrm{pH} 4.5]$ for 30 minutes at $37^{\circ} \mathrm{C}$, counterstained in fast green solution $(0.08 \%$ fast green, $1 \%$ acetic acid) or hematoxylin, and then air-dried, dipped in xylene and mounted. For Safranin O staining, rehydrated slides were incubated in Mayer's Hematoxylin No.2 for 10 minutes, Safranin O (0.1\%) for 5 minutes, and fast green solution for 1 minute and then air-dried, dipped in xylene, and mounted. For IHC, samples were collected after perfusion fixation with $10 \%$ neutral buffered formalin before being fixed for 24 hours and decalcified in 10\% EDTA (pH 7.4) for 10 days. Samples were paraffin embedded and cut into $4 \mu \mathrm{m}$ sections. Heat-mediated antigen retrieval was performed in $10 \mathrm{mM}$ sodium citrate (pH 6) at $60^{\circ} \mathrm{C}$ for 16 hours, followed by blocking in PBS with $1 \%$ BSA and $10 \%$ goat serum for 30 minutes. Sections were incubated with rabbit primary antibody diluted 1:50 for 3 hours at room temperature (JAK2, Cell Signaling Technologies, 3230; IGF1, Abcam, 63926) followed by incubation with goat anti-rabbit HRP polymer-linked secondary antibody (SignalStain Boost IHC Detection Reagent, Cell Signaling Technologies, 8114) for 30 minutes. Peroxidase detection was performed using the SignalStain DAB Substrate Kit (Cell Signaling Technologies, 8059) for 10 minutes, followed by counterstaining in hematoxylin. Staining controls with omission of primary antibody were performed, and no immunoreactivity was detected. For fluorescence imaging of reporter mice, euthanized mice were perfused with ice-cold $10 \%$ neutral buffered formalin; then, dissected tissues were further fixed for another 3 hours before being cryopreserved in PBS with 15\% sucrose for 1 hour and PBS with 15\% sucrose and 50\% OCT for 3 hours. They were then embedded in OCT medium. Knee joints from 1-week-old mice were embedded without decalcification. Frozen sections $(7 \mu \mathrm{m})$ were cut, washed in PBS, and mounted in fluroshield mounting medium with DAPI. Immunofluorescence images were obtained by a Zeiss inverted fluorescence microscope (Advanced Optical Microscopy Facility). For determination of growth plate size, histological sections were taken from the midsagittal plane of the proximal tibia, stained with Safranin O, and imaged with a light microscope. The ZP was defined as the area with columns of chondrocytes with a flattened appearance. The $\mathrm{ZH}$ was defined as the area where chondrocytes are enlarged, swollen, or vacuolated. The ZO was defined as the area with longitudinal spicules of cartilage surrounded by bone. As depicted in Figure 6C, smooth lines were drawn to delineate each zone by an operator who was blinded to the study groups. Measurements of the length of each zone, in the longitudinal plane of the bone, were made at the midpoint of each line using ImageJ software (NIH).

Cell culture. BM was collected from femurs and tibias of 4 -week-old mice and suspended in $\alpha$-MEM media (Thermo Fisher Scientific) supplemented with 10\% FBS (Sigma-Aldrich) and 100 units/mL penicillin/streptomycin (Thermo Fisher Scientific), passed through a $40 \mu \mathrm{m}$ cell strainer and treated with RBC lysis buffer containing ammonium chloride (Cell Signaling Technologies, 46232). BM cells were plated overnight in $\alpha$-MEM in a T175 flask. Nonadherent cells were collected, and $2.5 \times 10^{5}$ cells were plated in each well of a 24-well plate in $\alpha$-MEM supplemented with $100 \mathrm{ng} / \mathrm{mL}$ murine macrophage CSF (M-CSF; PeproTech, 315-02) for 3 days. For generation of BM-derived osteoclasts, cells were further incubated in $\alpha$-MEM with $100 \mathrm{ng} / \mathrm{mL} \mathrm{M}-\mathrm{CSF}$ and $100 \mathrm{ng} / \mathrm{mL}$ recombinant murine soluble receptor activator of NF- $\mathrm{KB}$ ligand (RANKL; PeproTech, 315-11C) for an additional 4 days (media changed on days 2 and 3), and the presence of multinucleated giant cells was confirmed by light microscopy. For generation of BM-derived macrophages, RANKL was omitted from cell cultures.

$q P C R$. Total RNA from cells and tissues was isolated using Trizol reagent (Invitrogen). For isolation of RNA from bone, femurs and tibias were cleaned of soft tissue, and BM was removed by centrifugation $\left(16,000 \mathrm{~g}\right.$ for 10 seconds at $\left.4^{\circ} \mathrm{C}\right)$. The distal third of the femur and proximal third of the tibia were then 
Table 2. Primer sequences for quantitative PCR

\begin{tabular}{|c|c|c|}
\hline Gene & Forward (5'-3') & Reverse (5'-3') \\
\hline $18 s$ & AGTCCСTGСССTTTCTACACA & CGATCCGAGGGCCTCACTA \\
\hline Acp5 & САСTCCСACСCTGAGATTTGT & СATCGTCTCCACGGTTCTG \\
\hline Calcr & GCAACGCTTTCACTTCTCAGA & GTTCCСACTGСATTСTCСACA \\
\hline Csk & TTCССTTCTGCAAAGGAGATGT & ACCAGGGCATAAGGCTGAGT \\
\hline Ctsk & GAAGAACACTCACCAGAAGCAG & TCCAGGTTATGGGCAGAGATT \\
\hline lgf1 & GGCATTGTGGATGAGTGTTG & TCTCCTTTGCAGCTTCGTTT \\
\hline Itgb3 & CCACACGAGGCGTGAACTC & CTTCAGGTTACATCGGGGTGA \\
\hline Jak2 & AGGCGACGGGAACAAGATGT & AGGCCATTCCCATCTAGAGC \\
\hline Mmp9 & CTGGACAGCCAGACACTAAAG & CTCGCGGCAAGTCTTCAGAG \\
\hline Nfatc1 & GACCCGGAGTTCGACTTCG & TGACACTAGGGGACACATAACTG \\
\hline Oscar & ССТАGССТСАТАСССССАG & CGTTGATCCCAGGAGTCACAA \\
\hline
\end{tabular}

placed in foil and pulverized under liquid nitrogen using a mortar and pestle. The samples were further homogenized in Trizol using Precellys Minilys Personal Tissue Homogenizer (Cayman Chemical). RNA was reverse transcribed with random primers using M-MLV enzyme (Invitrogen). qPCR was performed using specific primers and SYBR Green master mix on a 7900HT Fast-Real-Time PCR System (Applied Biosystems). The relative mRNA abundance of each gene was normalized to the expression level of $18 \mathrm{~S}$. $18 S$ expression was not significantly different between groups. Primer sequences are listed in Table 2.

Analysis of serum. Blood was collected at sacrifice by cardiac puncture. Serum IGF1 was measured using a mouse/rat IGF1 ELISA kit (R\&D Systems, MG100) or human IGF1 ELISA kit (R\&D Systems, DG100B) as per the manufacturer's instructions.

FACS. Control and $\mathrm{KO}$ mice were bred to the $m \operatorname{Tm} G$ reporter mouse to generate mice with $\mathrm{GFP}^{+}$ osteoclasts. Single-cell suspensions from long bones were isolated as previously described (40). Briefly, long bones were cleaned of soft tissue and flushed of marrow; they were then cut into $1 \mathrm{~mm}$ pieces. The fragments were digested twice for 30 minutes each in HBSS supplemented with $10 \mathrm{mg} / \mathrm{mL}$ type IV collagenase (Sigma-Aldrich, C5138) at $37^{\circ} \mathrm{C}$ with agitation. The solution was passed through a $70 \mu \mathrm{M}$ cell strainer to remove cell debris/clumps. Cell viability was verified by trypan blue staining and cells were suspended in FACS buffer at a concentration of $10 \mathrm{M} / \mathrm{mL}$. Osteoclasts were sorted based on the expression of GFP through the BD FACSAria-III Digital Cell Sorter (University Health Network Flow Cytometry Facility).

Statistics. For comparison of data between 2 groups, 2-tailed independent Student's $t$ test was performed. For comparison among multiple groups, 1-way ANOVA was performed. For comparison between 2 groups at different time points, 2-way ANOVA was performed. For comparison of weekly body weight between 2 groups, repeated-measures 1- or 2-way ANOVA was performed. Post hoc multiple comparisons were performed using the Holm-Sidak method. For normalized data, the residual method was employed where unstandardized residuals were calculated from linear regression and added to a constant. Data are presented as mean \pm SEM. Data were analyzed with SPSS version 20 (SPSS Inc.) and Prism GraphPad version 6. Statistical significance was defined as $P<0.05$.

Study approval. All experiments using mice in this study were approved by the Toronto General Hospital Research Institute Animal Care Committee (AUP 852).

\section{Author contributions}

DWD, WEW, and MW designed the research studies; DWD, JLY, JY, TS, SMS, SAS, EPT, YEL, and HL conducted the experiments; DWD analyzed the data; and DWD, WEW, and MW wrote and reviewed the manuscript.

\section{Acknowledgments}

This work was supported by operating grants from the Canadian Institute of Health Research (CIHR) MOP-142193 and PJT-159505 to MW. MW is supported by the Canada Research Chair in Signal Transduction in Diabetes Pathogenesis and the Soham and Shaila Ajmera Family Chair in Molecular Diabetes Research. WEW is supported by the Canada Research Chair in Bone and Muscle Development. DWD was 
supported by a Comprehensive Research Experience for Medical Students (CREMS) scholarship. Infrastructure support for the $\mu \mathrm{CT}$ system was provided by the Canadian Foundation for Innovation (WEW).

Address correspondence to: Minna Woo, 10-601D, Princess Margaret Cancer Research Tower, 101 College Street, Toronto, Ontario M5G 0A3, Canada. Phone: 416.581.7489; Email: mwoo@uhnresearch.ca.

1. Boyle WJ, et al. Osteoclast differentiation and activation. Nature. 2003;423(6937):337-342.

2. Tang P, et al. The role of microRNAs in osteoclasts and osteoporosis. RNA Biol. 2014;11(11):1355-1363.

3. Karmakar S, et al. Bone damage in rheumatoid arthritis: mechanistic insights and approaches to prevention. Rheum Dis Clin North Am. 2010;36(2):385-404.

4. Hienz SA, et al. Mechanisms of bone resorption in periodontitis. J Immunol Res. 2015;2015:615486.

5. Terpos E, et al. Pathogenesis of bone disease in multiple myeloma: from bench to bedside. Blood Cancer J. 2018;8(1):7.

6. Maurizi A, Rucci N. The osteoclast in bone metastasis: player and target. Cancers (Basel). 2018;10(7):218.

7. Rane SG, Reddy EP. Janus kinases: components of multiple signaling pathways. Oncogene. 2000;19(49):5662-5679.

8. Levine RL, et al. Role of JAK2 in the pathogenesis and therapy of myeloproliferative disorders. Nat Rev Cancer. 2007;7(9):673-683.

9. Veletic I, et al. Myelofibrosis osteoclasts are clonal and functionally impaired. Blood. 2019;133(21):2320-2324.

10. Murakami K, et al. A Jak1/2 inhibitor, baricitinib, inhibits osteoclastogenesis by suppressing RANKL expression in osteoblasts in vitro. PLoS One. 2017;12(7):0181126.

11. Kwak HB, et al. AG490, a Jak2-specific inhibitor, induces osteoclast survival by activating the Akt and ERK signaling pathways Mol Cells. 2008;26(5):436-442.

12. Winkeler CL, et al. Cathepsin K-Cre causes unexpected germline deletion of genes in mice. PLoS One. 2012;7(7):42005.

13. Crabtree NJ, et al. Dual-energy X-ray absorptiometry interpretation and reporting in children and adolescents: the revised 2013 ISCD Pediatric Official Positions. J Clin Densitom. 2014;17(2):225-242.

14. Ye L, et al. Osteopetrorickets due to Snx10 deficiency in mice results from both failed osteoclast activity and loss of gastric acid-dependent calcium absorption. PLoS Genet. 2015;11(3):1005057.

15. Dougall WC, et al. RANK is essential for osteoclast and lymph node development. Genes Dev. 1999;13(18):2412-2424.

16. Brooks AJ, Waters MJ. The growth hormone receptor: mechanism of activation and clinical implications. Nat Rev Endocrinol. 2010;6(9):515-525.

17. Sivasubramaniyam T, et al. Hepatic JAK2 protects against atherosclerosis through circulating IGF-1. JCI Insight. 2017;2(14):93735.

18. Shi SY et al. Hepatocyte-specific deletion of Janus kinase 2 (JAK2) protects against diet-induced steatohepatitis and glucose intolerance. J Biol Chem. 2012;287(13):10277-10288.

19. Sos BC, et al. Abrogation of growth hormone secretion rescues fatty liver in mice with hepatocyte-specific deletion of JAK2. J Clin Invest. 2011;121(4):1412-1423.

20. Jiao Y et al. Mex3c regulates insulin-like growth factor 1 (IGF1) expression and promotes postnatal growth. Mol Biol Cell. 2012;23(8):1404-1413.

21. Wu Y, et al. Elevated levels of insulin-like growth factor (IGF)-I in serum rescue the severe growth retardation of IGF-I null mice. Endocrinology. 2009;150(9):4395-4403.

22. Glatt V, et al. Age-related changes in trabecular architecture differ in female and male C57BL/6J mice. J Bone Miner Res. 2007;22(8):1197-1207.

23. Lee Y, Kim H. The role of Jak/STAT pathways in osteoclast differentiation. Biomol Ther. 2011;19(2):141-148.

24. Nishiyama K, et al. Stimulatory effect of growth hormone on bone resorption and osteoclast differentiation. Endocrinology. 1996;137(1):35-41.

25. Duplomb L, et al. Interleukin-6 inhibits receptor activator of nuclear factor kappaB ligand-induced osteoclastogenesis by diverting cells into the macrophage lineage: key role of Serine 727 phosphorylation of signal transducer and activator of transcription 3 . Endocrinology. 2008;149(7):3688-3697.

26. Nagata N, et al. Inhibition of RANKL-induced osteoclast formation in mouse bone marrow cells by IL-12: involvement of IFN-gamma possibly induced from non-T cell population. Bone. 2003;33(4):721-732.

27. Lee Y, et al. The ubiquitin-mediated degradation of Jak1 modulates osteoclastogenesis by limiting interferon-beta-induced inhibitory signaling. Blood. 2008;111(2):885-893.

28. Wang LH, et al. JAK3, STAT, and MAPK signaling pathways as novel molecular targets for the tyrphostin AG-490 regulation of IL-2-mediated T cell response. J Immunol. 1999;162(7):3897-3904.

29. Stratikopoulos E, et al. The hormonal action of IGF1 in postnatal mouse growth. Proc Natl Acad Sci U S A. 2008;105(49):19378-19383.

30. Sjögren K, et al. Liver-derived insulin-like growth factor I (IGF-I) is the principal source of IGF-I in blood but is not required for postnatal body growth in mice. Proc Natl Acad Sci U S A. 1999;96(12):7088-7092.

31. Yakar S, et al. Normal growth and development in the absence of hepatic insulin-like growth factor I. Proc Natl Acad Sci U S A 1999;96(13):7324-7329.

32. Govoni KE, et al. Disruption of insulin-like growth factor-I expression in type IIalphaI collagen-expressing cells reduces bone length and width in mice. Physiol Genomics. 2007;30(3):354-362.

33. Govoni KE, et al. Conditional deletion of insulin-like growth factor-I in collagen type 1alpha2-expressing cells results in postnatal lethality and a dramatic reduction in bone accretion. Endocrinology. 2007;148(12):5706-5715.

34. Lau KH, et al. Osteocyte-derived insulin-like growth factor I is essential for determining bone mechanosensitivity. Am J Physiol Endocrinol Metab. 2013;305(2):E271-E281.

35. Wang J, et al. Igf1 promotes longitudinal bone growth by insulin-like actions augmenting chondrocyte hypertrophy. FASEB J. 1999;13(14):1985-1990.

36. Nakamura T, et al. Estrogen prevents bone loss via estrogen receptor alpha and induction of Fas ligand in osteoclasts. Cell. 
2007;130(5):811-823

37. Wagner KU, et al. Impaired alveologenesis and maintenance of secretory mammary epithelial cells in Jak2 conditional knockout mice. Mol Cell Biol. 2004;24(12):5510-5520

38. Muzumdar MD, et al. A global double-fluorescent Cre reporter mouse. Genesis. 2007;45(9):593-605.

39. Liu JL, et al. Insulin-like growth factor-I affects perinatal lethality and postnatal development in a gene dosage-dependent manner: manipulation using the Cre/loxP system in transgenic mice. Mol Endocrinol. 1998;12(9):1452-1462.

40. Wang W, et al. Osteoblast sorting and intracellular staining of CXCL12. Bio Protoc. 2018;8(10):2858. 